\title{
Feshbach-Fano approach for calculation of Auger decay rates using equation-of-motion coupled-cluster wave functions: Numerical examples and benchmarks
}

\author{
Wojciech Skomorowski* and Anna I. Krylov ${ }^{\dagger}$ \\ Department of Chemistry, University of Southern California, Los Angeles, California 90089, USA
}

\begin{abstract}
X-ray photon absorption leads to the creation of highly excited species, which often decay through the Auger process. The theoretical treatment of Auger decay is challenging because of the resonance nature of the initial core-excited or core-ionized states and the continuous nature of the ejected electron. In a companion paper (Skomorowski, Krylov, submitted to J. Chem. Phys.), we have introduced a theoretical framework for computing Auger rates based on the Feshbach-Fano approach and the equation-of-motion coupled-cluster ansätze augmented with core-valence separation. The outgoing Auger electron is described with a continuum orbital. We considered two approximate descriptions - a plane wave and a Coulomb wave with an effective charge. Here we use the developed methodology to calculate Auger transition rates in core-ionized and core-excited benchmark systems $\left(\mathrm{Ne}, \mathrm{H}_{2} \mathrm{O}, \mathrm{CH}_{4}\right.$, and $\mathrm{CO}_{2}$ ). Comparison with available experimental spectra shows that the proposed computational scheme provides reliable $a b$ initio predictions of the Auger spectra. The reliability, cost-efficiency, and robust computational setup of this methodology offer advantages in applications to a large variety of systems.
\end{abstract}

\section{INTRODUCTION}

By providing tunable high-energy radiation, advanced light sources enable a variety of X-ray based spectroscopies[1-3]. Recent advances in beam quality greatly expanded the possible applications of $\mathrm{X}$ rays, giving rise to a proliferation of techniques including those operating in time-resolved and nonlinear regimes[1-5]. Fundamentally, these spectroscopies exploit electronic transitions involving core orbitals, whose localized nature enables the local environment to be probed, thus providing complementary information to valence-based techniques.

As in the case of VUV-based techniques[6], theoretical modeling is required to relate experimental measurements to molecular structures. Consequently, experimental advances have stimulated the development of theoretical techniques for core-level transitions[5]. Owing to their special properties, accurate description of core-level states is much more challenging than description of valence states[5]. Despite significant progress in extending quantum chemistry to core-level states, theoretical tools for modeling X-ray spectroscopies are still lagging behind experimental capabilities, creating a bottleneck for maximizing the scientific impact of advanced light sources facilities.

Recently, many quantum chemistry methods have been adapted to treat core-level states by utilizing the core-valence separation (CVS) [7] scheme, which decouples highly-excited core-level states from the continuum of valence excitations and allows one to describe

\footnotetext{
* skomorow@usc.edu

$\dagger$ krylov@usc.edu
}

metastable core-level states by $\mathcal{L}^{2}$-integrable wave functions. By using CVS, many-body methods developed for bound ionized and excited states have been adapted to treat highly energetic unbound (with respect to electron ejection) core-level states. In particular, equation-of-motion coupled-cluster (EOM-CC) methods[8-11] have been extended to model core ionization and core excitation processes, including non-linear regimes[12-21]. The benchmarks and applications illustrated the excellent performance of CVS-enabled EOMCC methods[14, 21-25]. Importantly, in all these extensions, the continuum was simply ignored. Consequently, such calculations are only able to deliver the energies of the core-level states and intensities of the spectral transitions. The lifetimes of the core-level states and the respective broadening of the spectral lines could not be computed. In our companion paper[26], we presented the extension of the theory to compute the decay rates of the core-level states.

The core-level states are Feshbach resonances that can decay by a two-electron process, called Auger decay, and its non-local variants - intermolecular Coulomb decay and electron-transfer mediated decay. As illustrated in Fig. 1, Auger decay is a process in which an $N$ electron highly excited core-level state decays into an $N$ 1 electron valence state and a free electron. The theoretical framework, described in the companion paper[26], is based on the Feshbach-Fano treatment of resonance phenomena. In this approach, the full function space is artificially divided into the bound and unbound spaces, and the resonances are described in terms of the interacting diabatic-like states, representing the bound part of the resonance and the continuum decay channels, respectively. The essential feature of our formulation of the Feshbach-Fano treatment is the use of CVS to define the Feshbach projectors. We use EOM-CC to de- 


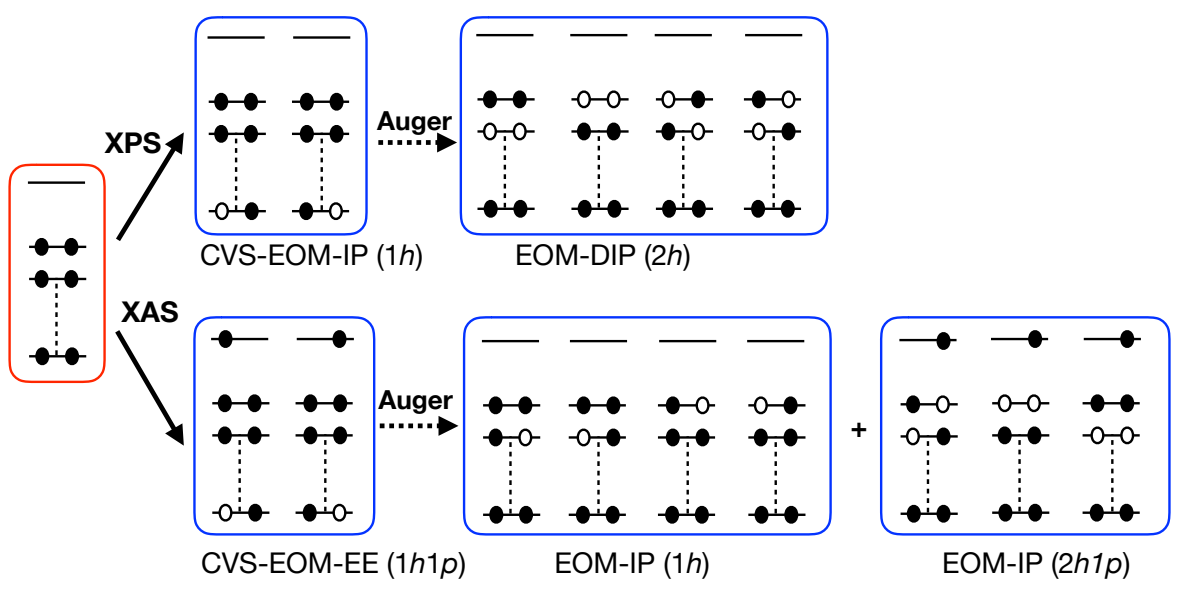

FIG. 1. Initial and target states corresponding to different types of Auger effect. Top row illustrates regular Auger decay, which is relevant for X-ray photoionization spectroscopy (XPS). The initial and decay states can be described by CVS-EOMIP and EOM-DIP, respectively. The bottom row illustrates resonant Auger decay, which is relevant to X-ray absorption spectroscopy (XAS). The initial state in resonant Auger decay can be described by CVS-EOM-EE. The decay states can be described by EOM-IP-CCSD. In resonant participator decay, the initially excited electron is ejected-hence, the decay states are described by $1 h$ EOM-IP operators. In resonant spectator decay, the initially excited electron remains and another electron is ejected, leading to excited states of the ion that require $2 h 1 p$ EOM-IP operators.

scribe the bound part of the wave function in the initial and final states of the Auger decay and use continuum orbitals to represent the Auger electrons. The versatility of the EOM-CC ansatz facilitates efficient treatment of complex, multi-configurational and open-shell wave functions, as well as systematic inclusion of electron correlation.

To describe the decay channels, we combine manyelectronic states described by EOM-CCSD with a continuum orbital. In this work, we approximate the continuum orbital by a plane wave or a Coulomb wave, which avoids numerical integration in the calculations of mixed bound-continuum electron-repulsion integrals. The working equations for the calculations of the partial autoionization widths are expressed in terms of oneand two-body Dyson functions[27, 28], contracted with the bound-continuum integrals.

In this paper, we illustrate the performance of the new methodology by calculating Auger decay rates in several well-characterized systems. Specifically, we considered non-resonant Auger effect due to a single $K$ shell vacancy in Ne atom; $\mathrm{H}_{2} \mathrm{O}, \mathrm{CH}_{4}$, and $\mathrm{CO}_{2}$ (C-edge) molecules; and resonant Auger effect in core-excited $\mathrm{Ne}$ $(1 s \rightarrow 3 p)$ and $\mathrm{CO}_{2}\left(\mathrm{C} 1 s \rightarrow \pi_{u}^{\star}\right)$.

\section{COMPUTATIONAL DETAILS}

Within our approach, the calculations of Auger decay rates require two essential components: twobody Dyson functions and mixed bound-continuum two-electron integrals. Two-body Dyson functions are bound-domain properties and they are calculated from appropriate EOM-CC solutions, as illustrated in Fig. 1. All systems in this study have closed-shell ground states, which served as reference states in the CCSD and EOM-CCSD computations. Initial states for nonresonant Auger decay were obtained from CVS-EOMIP-CCSD (removal of one core electron), whereas the final states (decay channels) were computed by means of EOM-DIP-CCSD (removal of two valence electrons). For resonant Auger decay the initial state was computed with CVS-EOM-EE-CCSD (excitation of core electron to a valence shell), whereas the corresponding final states were described with EOM-IP-CCSD (removal of one valence electron). Core electrons were frozen in all valence calculations and treated in accordance to the fc-CVS prescription[14] in the calculations of corelevel states. Frozen core was defined as comprising $K$-shells on $\mathrm{Ne} / \mathrm{O} / \mathrm{C}$ atoms. Unless otherwise stated, in bound-state calculations we employed fully uncontracted $6-311+\mathrm{G}(3 \mathrm{df})$ basis set $[29,30]$ for all atoms. As has been recently shown [22], this one-electron basis offers a good balance between cost and accuracy for core ionized/excited states. The calculations were carried out using MP2/aug-cc-pVTZ optimized structures; the respective Cartesian coordinates are given in the Supplementary Information (SI).

Here, we test two simple approaches for the continuum orbital: a plane wave, and a Coulomb wave expanded in terms of auxiliary basis set of the products of Gaussian and plane wave functions (GPW). The details of the implementation of mixed GTO-GPW integrals are described in the companion paper[26]. As for the Coulomb wave, the expansion coefficients in terms of the GPW functions were generated following the pseudo- 
partial wave method of Szczygieł et al. [31]. The parameters of the auxiliary GPW basis set for each energy and pseudo-partial wave were determined through the optimization by means of the differential evolution algorithm. For the optimization procedure the reference values of the Coulomb wave were generated on a radial grid from 0 to 2 bohr with a spacing of $0.05 \mathrm{bohr}$ and from 2 to 10 bohr with a spacing of 0.1 bohr. Because Auger decay is a rather local phenomenon, with core orbitals primary contributing to the effect, there is no need for more extended representation of the continuum orbital in the calculations of two-electron integrals. Based on the numerical tests, we truncate the pseudopartial wave expansion at $l_{\max }=6$, with each pseudopartial wave represented in terms of $n=10$ Gaussian components. The choice of the effective charge for the Coulomb wave is discussed below.

All electronic structure calculations were carried out with the $Q$-Chem package $[32,33]$. The optimization of the GPW basis set for Coulomb wave expansion was performed with the Mathematica package [34].

\section{RESULTS AND DISCUSSION}

\section{A. $\mathrm{Ne}\left(1 s^{-1}\right)$}

$K$-shell vacancy in Ne atom has been, arguably, the most frequently studied example of the Auger effect, both experimentally and theoretically [35, 37-41]. The Auger spectrum in $\mathrm{Ne}^{+}\left(1 s^{-1}\right)$ is particularly suitable for in-depth analysis as it is features sharp discrete lines with no vibrational broadening or vibronic coupling, with easy to identify diagram (main) transitions and satellite structure. Therefore, the $\mathrm{Ne}^{+}\left(1 s^{-1}\right)$ state is often used as a test case to validate various theoretical approaches for Auger rates. In the Ne $K-L L$ Auger spectrum there are five main lines, corresponding to the formation of residual $\mathrm{Ne}^{2+}$ ion in the following states: ${ }^{1} \mathrm{D}\left(2 p^{-2}\right),{ }^{1} \mathrm{~S}\left(2 p^{-2}\right),{ }^{3} \mathrm{P}\left(2 s^{-1} p^{-1}\right),{ }^{1} \mathrm{P}\left(2 s^{-1} 2 p^{-1}\right)$, and ${ }^{1} \mathrm{~S}\left(2 s^{-2}\right)$. Branching ratios for populating those five channels have been reported a few times in the literature, with high consistency of the results [35]. This is in contrast with a significant uncertainty in the experimental determination of the total natural width of the $\mathrm{Ne}^{+}\left(1 s^{-1}\right)$ level. The reported experimental natural linewidths vary in the range from roughly 0.21 to $0.30 \mathrm{eV}[37,42-45]$. For the purpose of the present work, we refer to the numbers reported in a recent high-precision spectroscopic study on $\mathrm{Ne}^{+}$ions near the $K$-edge [37]. Based on measured photo-ionization cross sections, the authors determined the total natural width of the $\mathrm{Ne}^{+}\left(1 s^{-1}\right)$ level to be $261 \pm 5 \mathrm{meV}$, which includes contribution from both radiative and Auger decay, and the latter can be further split into one, two, and threeelectron emission channels. Here we focus exclusively on one-electron Auger decay channels, for which par- tial width can be estimated to be $242 \pm 10 \mathrm{meV}$, based on relative photoionization cross-sections and resonance strengths reported in Table 5/6 of Ref. [37].

Table I shows energies for the main Ne $K-L L$ Auger channels, calculated with various one-electron basis set, and compared to the experimental values from Ref. [35]. In accordance with the results of benchmark study[22], the relatively compact $6-311+\mathrm{G}(3 \mathrm{df}$ ) basis set (fully uncontracted) provides energies of the same quality as more sizable Dunning-type bases. Our calculated energies differ from the experimental ones by 0.5 to $\sim 1.5 \mathrm{eV}$, which is within error bars of the method[14]. The remaining errors are due to the neglect of the relativistic contributions [21], higher order correlation effects, and incomplete one-electron basis set. The energies reported in Table I neglect also the resonance shift, a contribution which is relatively expensive to calculate. It is known that for Auger resonances the energy shift $\Delta_{n}$ is a minor correction (usually $<0.2 \mathrm{eV}[46]$ ), and thus it is well within the error of our main energy term $E_{n}$.

Tables II and III present absolute values and branching ratios for Ne $K-L L$ Auger transition rates, obtained with different models for the continuum orbital, and compared with the most accurate experimental results. The experimental values for partial widths reported in the last column of Table II are obtained from the analysis of the results from Refs. [35, 37], where we have assumed the total one-electron Auger width of $242 \pm 10 \mathrm{meV}$ from Ref. [37] and the branching ratios from Ref. [35].

As noted above, we employed a simple model for the continuum orbital, either a plane wave or a Coulomb wave. Coulomb wave requires to specify an effective charge of the residual ion. Depending on the modeled properties, several protocols for estimating optimal effective charges have been proposed. We have tested here three different models to generate optimal effective charges. The first and the simplest model is to employ Slater rules [47], which provide universal screening constants for each atomic shell. Based on the Slater rules, residual ion $\mathrm{Ne}^{2+}$ induces an effective charge equal to 6.2. Slater rules do not account for differences between sub-shells so they predict the same screening due to $2 \mathrm{~s}$ and $2 p$ electrons. This limitations has been lifted in a couple of other studies aiming to determine optimal screening constants [36, 48, 49].

Here, we tested modified Slater rules and resulting effective charges based on the approach shown in Ref. [36]. In this work the optimal screening constants were generated by fitting energies of multiple atomic and ionic states with primary configuration of the type $1 s^{2} 2 s^{m} 2 p^{n}$. By including explicitly the ionic configurations in the fit this model reflects better the physics we aim to describe - i.e., the outgoing electron moving in the field of the $\mathrm{Ne}^{2+}$ ion. We refer to the effective charges based on Ref. [36] as 'updated Slater' in Tables II and III. 
TABLE I. Auger electron energies (in eV) corresponding to $K-L L$ transitions in core-ionized neon atom ${ }^{a}$.

\begin{tabular}{l|c|c|c|c}
\hline \hline Channel & u-6-311+G(3df) & u-aug-cc-pVTZ & u-d-aug-cc-pVTZ & Exp-t [35] \\
\hline${ }^{1} \mathrm{D}\left(2 p^{-2}\right)$ & 803.62 & 803.74 & 803.74 & $804.30(8)$ \\
${ }^{1} \mathrm{~S}\left(2 p^{-2}\right)$ & 800.09 & 800.21 & 800.21 & $800.60(8)$ \\
${ }^{3} \mathrm{P}\left(2 s^{-1} 2 p^{-1}\right)$ & 781.19 & 781.24 & 781.24 & $782.24(8)$ \\
${ }^{1} \mathrm{P}\left(2 s^{-1} 2 p^{-1}\right)$ & 770.50 & 770.56 & 770.56 & $771.71(8)$ \\
${ }^{1} \mathrm{~S}\left(2 s^{-2}\right)$ & 746.55 & 746.54 & 746.54 & $748.14(8)$ \\
\hline \hline
\end{tabular}

TABLE II. Partial and total Auger decay widths (in meV) for $K-L L$ transitions in core-ionized neon atom. Calculations used either a plane wave or Coulomb wave (with specified effective charges) to model the outgoing electron.

\begin{tabular}{|c|c|c|c|c|c|}
\hline Channel & $\begin{array}{c}\text { Plane wave } \\
\left(Z_{\text {eff }}=0\right)\end{array}$ & $\begin{array}{c}\text { Slater } \\
\left(Z_{e f f}=6.2\right)\end{array}$ & Updated Slater $^{\mathrm{a}}$ & $Z_{e f f} \sim{\sqrt{1 /\left\langle r^{2}\right\rangle}}^{\mathrm{b}}$ & Exp- $t^{c}$ \\
\hline${ }^{1} \mathrm{D}\left(2 p^{-2}\right)$ & 154.9 & 105.5 & 109.8 & 117.5 & $147 \pm 6$ \\
\hline${ }^{1} \mathrm{~S}\left(2 p^{-2}\right)$ & 4.4 & 12.2 & 12.2 & 10.5 & $23 \pm 1$ \\
\hline${ }^{3} \mathrm{P}\left(2 s^{-1} 2 p^{-1}\right)$ & 116.5 & 19.2 & 15.0 & 23.8 & $15 \pm 1$ \\
\hline${ }^{1} \mathrm{P}\left(2 s^{-1} 2 p^{-1}\right)$ & 4.8 & 41.5 & 43.0 & 39.2 & $42 \pm 2$ \\
\hline${ }^{1} \mathrm{~S}\left(2 s^{-2}\right)$ & 19.0 & 28.0 & 23.1 & 27.1 & $15 \pm 1$ \\
\hline Total & 299.6 & 206.4 & 203.1 & 218.1 & $242 \pm 10$ \\
\hline
\end{tabular}

${ }^{\text {a }}$ Based on screening constants optimized for ionic configurations (OHAO model from Ref. [36]): $Z_{e f f}=5.8 / 5.8 / 6.8 / 6.8 / 7.8$ for respective channels.

b Based on the size of the vacant valence orbitals: $Z_{e f f}=4.9 / 4.9 / 5.7 / 5.7 / 6.6$ for respective channels. For $2 s^{-1} 2 p^{-1}$ configuration the geometrical average is applied.

${ }^{c}$ Experimental data [35, 37] derived from Refs. [35, 37] (see text for the explanations).

Following a different approach, one can obtain an effective charge based on the size of the valence orbital that is being vacated in the Auger decay. To this end, one can employ the relation for the hydrogen-like ion:

$$
\left\langle r^{2}\right\rangle=\frac{n^{2}}{2 Z_{\text {eff }}^{2}}\left[5 n^{2}+1-3\left\langle l^{2}\right\rangle\right]
$$

which links the size of the orbital $\left\langle r^{2}\right\rangle$ with its effective charge $Z_{e f f}$, principle quantum number $n$, and angular momentum $\left\langle l^{2}\right\rangle$. For an atom, all requisite quantities for computing $Z_{\text {eff }}$ from this equation are easily obtainable. As expected, both the updated Slater rules and $\left\langle r^{2}\right\rangle$ approach predict larger screening due to $2 s$ than $2 p$ electrons, and, consequently, a smaller effective charge for $2 p^{-2}$ than for $2 s^{-2}$ configurations of $\mathrm{Ne}^{2+}$. The exact values of the effective charges for all channels within each model are given in the footnote of Table II.

As one can see from Tables II and III a plane wave continuum orbital leads to rather poor results for Auger partial widths and branching ratios, although the total width is reasonable and the channel with the largest intensity $\left({ }^{1} \mathrm{D}\right)$ is identified correctly. The results can be significantly improved when a Coulomb wave with rational effective charge is employed to model the free electron. Indeed, all tested sets of the effective charges correct absolute and relative intensities for ${ }^{1} \mathrm{P}$ and ${ }^{3} \mathrm{P}$ channels, with ${ }^{1} \mathrm{D}$ remaining the dominant channel. In contrast, the results for the two ${ }^{1} \mathrm{~S}$ channels do not show much improvement with any of the tested Coulomb waves. To investigate this further, Fig. 2 shows how each partial width changes as a function of the Coulomb wave charge $Z_{\text {eff }}$. One can see that for ${ }^{1} \mathrm{D},{ }^{1} \mathrm{P}$ and ${ }^{3} \mathrm{P}$ one can pick $Z_{\text {eff } f}$ such that the calculated widths match the experimental values. Also, as expected, the optimal $Z_{\text {eff }}$ for ${ }^{1} \mathrm{D}$ would be smaller than for ${ }^{1} \mathrm{P}$ and ${ }^{3} \mathrm{P}$ channels. In contrast, there is no rational $Z_{e f f}$, which would allow to adjust theoretical widths for both ${ }^{1} \mathrm{~S}$ channels. This is an illustration of the importance of the inter-channel coupling for the $K-L L$ Auger decay in Ne. As was shown previously[38], the two ${ }^{1} \mathrm{~S}$ channels strongly mix with each other and this coupling leads to the redistribution of the Auger intensities between the two channels. Within the independent channel model, the present calculations cannot reproduce the effect of the channel mixing. At the same time, the inter-channel coupling does not affect the sum of the two ${ }^{1} \mathrm{~S}$ intensities, and this sum is reproduced accurately by all Coulomb wave models from Table II. As far as the total Auger width is concerned, the last tested model with effective charges based on the orbital size gives the best number, which is still, however, $\sim 20 \mathrm{meV}$ off the experimental value. This discrepancy is mostly due to significantly underestimated the Auger rate for the ${ }^{1} \mathrm{D}$ channel, and this error can be traced back to deficiency of the single-channel calculations. As it was shown [39], 
TABLE III. Relative widths (given in \%) for $K-L L$ transitions in core-ionized neon atom ${ }^{a}$.

\begin{tabular}{l|c|c|c|c|c}
\hline \hline Channel & $\begin{array}{c}\text { Plane wave } \\
\left(Z_{e f f}=0\right)\end{array}$ & $\begin{array}{c}\text { Slater } \\
\left(Z_{e f f}=6.2\right)\end{array}$ & Updated Slater [36] & $Z_{e f f} \sim \sqrt{1 /\left\langle r^{2}\right\rangle}$ & Exp-t [35] \\
\hline${ }^{1} \mathrm{D}\left(2 p^{-2}\right)$ & 51.7 & 51.1 & 54.1 & 53.9 & 60.9 \\
${ }^{1} \mathrm{~S}\left(2 p^{-2}\right)$ & 1.5 & 5.9 & 6.0 & 4.8 & 9.5 \\
${ }^{3} \mathrm{P}\left(2 s^{-1} 2 p^{-1}\right)$ & 38.9 & 9.3 & 7.4 & 10.9 & 6.3 \\
${ }^{1} \mathrm{P}\left(2 s^{-1} 2 p^{-1}\right)$ & 1.6 & 20.1 & 21.2 & 18.0 & 17.2 \\
${ }^{1} \mathrm{~S}\left(2 s^{-2}\right)$ & 6.3 & 13.6 & 11.4 & 12.4 & 6.1 \\
\hline \hline
\end{tabular}

${ }^{a}$ Calculations with the same models for the outgoing electron as in Table II.
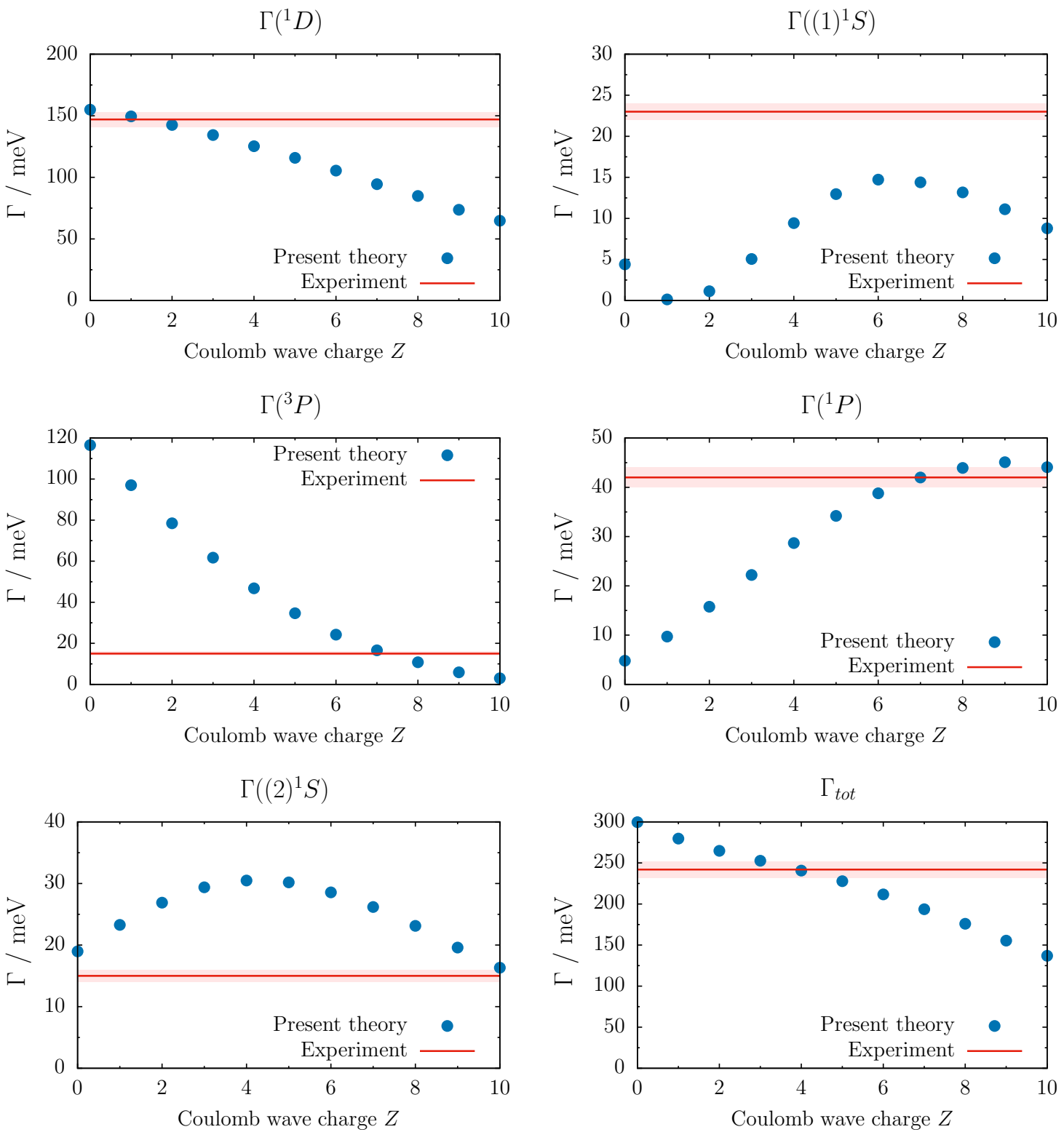

FIG. 2. Theoretical partial and total Auger decay widths for $K-L L$ transitions in core-ionized Ne as a function of Coulomb wave charge, compared with experimental data [35, 37]. 
multi-channel approach increases the ${ }^{1} \mathrm{D}$ rate by around $10 \%$ when compared to the single-channel rate. Overall, the simple model for the continuum orbital based on a Coulomb wave and rational effective charge is able to reproduce the main features and relative intensities of the Ne $K-L L$ Auger spectrum fairly well, which validates our approach. To resolve the remaining discrepancies, it is necessary to include the interaction between the continuum channels, which is beyond the scope of the present study.

Before discussing the results for other benchmark systems, let us address the convergence of the calculated widths with respect to the details of the computational protocol. The first question is the convergence with respect to one-electron basis set used for the bound domain electronic structure calculations. The Auger widths for Ne $K-L L$ obtained with three one-electron basis sets are shown in SI, and they all agree with each other very well. The convergence for the widths is as good as for the energies (see Table I). This is a simple consequence of the fact that within our approach the one-electron basis set only affects two-body Dyson functions (i.e., properties purely from the bound domain), whereas the continuum orbital is separated from the bound domain and it is described with a different function. Therefore, it is more important to address convergence with respect to the parameters used to model the continuum orbital. While the representation of a plane wave does not involve approximations, for a Coulomb wave we employ the auxiliary basis set and the pseudopartial wave expansion. Figure 3 shows how the partial widths converge with respect to the pseudo-partial waves (for $Z_{\text {eff }}=6.2$ ). For all channels the convergence is pretty fast and already for $l_{\max }=6$ the results are essentially converged. This is encouraging, given that the scattering energies considered here are of the order of $800 \mathrm{eV}$ and standard partial wave expansion would require many more terms.

\section{B. $\mathbf{H}_{2} \mathbf{O}\left(1 s^{-1}\right)$}

Similarly to the Ne atom, the Auger electron spectrum due to a single $K$-shell vacancy in a water molecule has been the subject of extensive studies, both theoretical and experimental [50-56]. Numerous theoretical investigations revealed the effects of nuclear dynamics, electronic correlation, and satellite transitions in the Auger profile of $\mathrm{H}_{2} \mathrm{O}[52,54,56]$. Decay of the single core vacancy in $\mathrm{H}_{2} \mathrm{O}$ is of the $K-L L$ type, and, to a large extent, it is reminiscent of the corresponding $K-L L$ decay in the isoelectronic Ne atom. However, the lower symmetry of $\mathrm{H}_{2} \mathrm{O}$ results in a large number of distinct final channels with two vacancies in the valence shell. Additionally, Franck-Condon broadening leads to a broad spectral profile, in contrast to discrete lines observed in the Auger spectrum of atomic neon. A

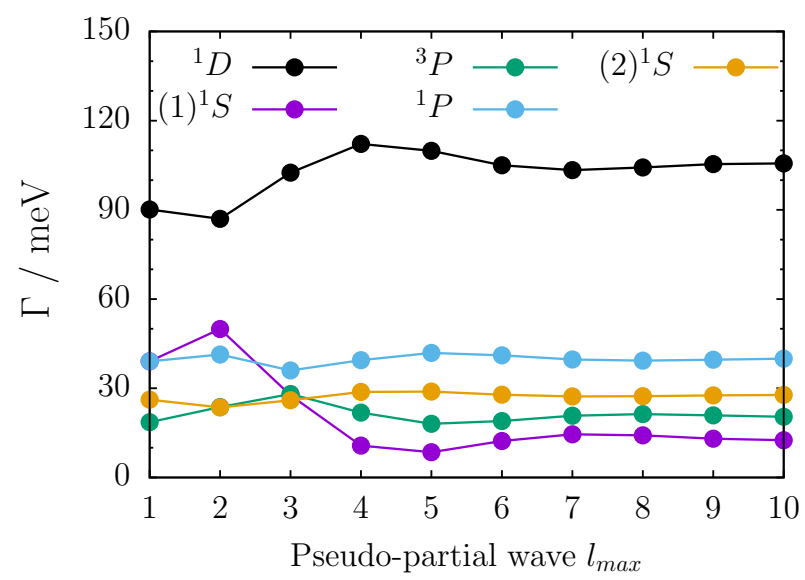

FIG. 3. Convergence of partial decay widths for $K-L L$ transitions in core-ionized $\mathrm{Ne}$ with respect to pseudo-wave expansion of the Coulomb wave function. Calculations were done with $Z_{\text {eff }}=6.2$.

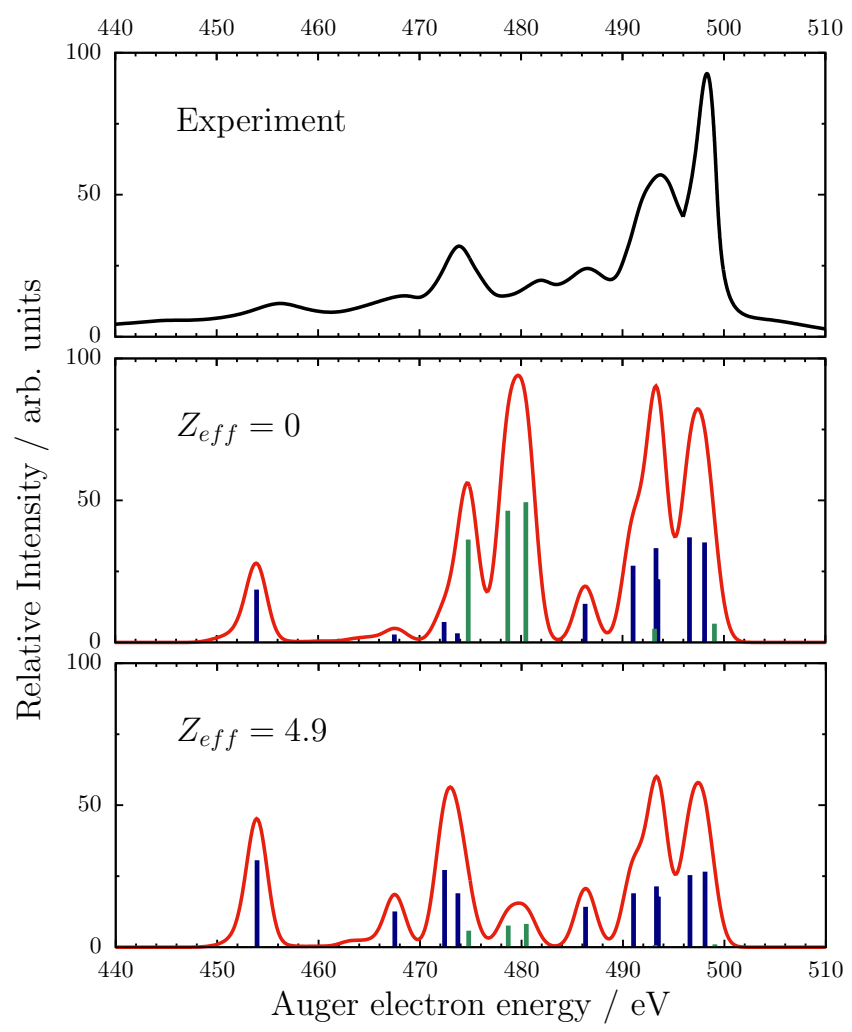

FIG. 4. Experimental [50] and computed Auger spectra for singly ionized water molecule. Middle and bottom panels show the calculations with a plane wave and a Coulomb wave, respectively. Theoretical curves obtained from the stick spectra by assuming constant Gaussian broadening of 1.0 eV FWHM. Dark-blue sticks correspond to singlet channels while green sticks to triplet channels, respectively. 
TABLE IV. Energies (in eV), total $\Gamma_{\text {tot }}$ and partial widths $\Gamma_{i}$ (in meV) for the main channels in the Auger decay of $K$-shell ionized water. Calculations of the widths used either a plane wave or a Coulomb wave with effective charge $Z_{\text {eff }}=4.9$ for all channels.

\begin{tabular}{|c|c|c|c|c|}
\hline \multirow{2}{*}{ Channel } & \multirow{2}{*}{ Energy / eV } & \multicolumn{3}{|c|}{$\Gamma_{i} / \mathrm{meV}$} \\
\hline & & $Z_{e f f}=0$ & $Z_{e f f}=4.9$ & Theory from Ref. [56] \\
\hline${ }^{3} \mathrm{~B}_{1}\left(3 a_{1}^{-1} 1 b_{1}^{-1}\right)$ & 499.07 & 3.3 & 0.5 & 0.4 \\
\hline${ }^{1} \mathrm{~A}_{1}\left(1 b_{1}^{-2}\right)$ & 498.09 & 17.6 & 13.3 & 19.0 \\
\hline${ }^{1} \mathrm{~B}_{1}\left(3 a_{1}^{-1} 1 b_{1}^{-1}\right)$ & 496.61 & 18.5 & 12.7 & 18.0 \\
\hline${ }^{3} \mathrm{~A}_{2}\left(1 b_{1}^{-1} 1 b_{2}^{-1}\right)$ & 495.07 & 0.0 & 0.0 & 0.0 \\
\hline${ }^{1} \mathrm{~A}_{1}\left(3 a_{1}^{-2}\right)$ & 493.49 & 11.1 & 8.9 & 13.1 \\
\hline${ }^{1} \mathrm{~A}_{2}\left(1 b_{1}^{-1} 1 b_{2}^{-1}\right)$ & 493.31 & 16.6 & 10.7 & 15.2 \\
\hline${ }^{3} \mathrm{~B}_{2}\left(3 a_{1}^{-1} 1 b_{2}^{-1}\right)$ & 493.17 & 2.4 & 0.4 & 0.3 \\
\hline${ }^{1} \mathrm{~B}_{2}\left(3 a_{1}^{-1} 1 b_{2}^{-1}\right)$ & 491.04 & 13.5 & 9.5 & 13.2 \\
\hline${ }^{1} \mathrm{~A}_{1}\left(1 b_{2}^{-2}\right)$ & 486.32 & 6.8 & 7.1 & 9.8 \\
\hline${ }^{3} \mathrm{~B}_{1}\left(2 a_{1}^{-1} 1 b_{1}^{-1}\right)$ & 480.47 & 24.7 & 4.1 & 3.0 \\
\hline${ }^{3} \mathrm{~A}_{1}\left(2 a_{1}^{-1} 3 a_{1}^{-1}\right)$ & 478.69 & 23.2 & 3.8 & 2.6 \\
\hline${ }^{3} \mathrm{~B}_{2}\left(2 a_{1}^{-1} 1 b_{2}^{-1}\right)$ & 474.79 & 18.1 & 2.9 & 1.6 \\
\hline${ }^{1} \mathrm{~B}_{1}\left(2 a_{1}^{-1} 1 b_{1}^{-1}\right)$ & 473.73 & 1.6 & 9.5 & 10.0 \\
\hline${ }^{1} \mathrm{~A}_{1}\left(2 a_{1}^{-1} 3 a_{1}^{-1}\right)$ & 472.42 & 3.6 & 13.6 & 11.0 \\
\hline${ }^{1} \mathrm{~B}_{2}\left(2 a_{1}^{-1} 1 b_{2}^{-1}\right)$ & 467.51 & 1.4 & 6.3 & 6.6 \\
\hline${ }^{1} \mathrm{~A}_{1}\left(2 a_{1}^{-2}\right)$ & 453.93 & 9.3 & 15.3 & 4.1 \\
\hline$\Gamma_{\text {tot }}$ & & 175.1 & 121.7 & 145.6 \\
\hline
\end{tabular}

simple molecular orbital picture permits to divide main Auger transitions in $\mathrm{H}_{2} \mathrm{O}$ into three regions: (1) from $\sim 500$ to $\sim 485 \mathrm{eV}$, where two electrons are removed from the outer-valence shell $\left(3 a_{1} 1 b_{1} 1 b_{2}\right),(2)$ from $\sim 480$ to $\sim 460 \mathrm{eV}$, where one electrons is removed from the outer-valence shell and one from the inner valence shell $\left(2 a_{1}\right)$, and $(3)$ from $\sim 460$ to $\sim 450 \mathrm{eV}$, where two electron are removed from the inner-valence shell $\left(2 a_{1}\right)$. As can be seen from the experimental Auger spectrum (the upper panel of Fig. 4) the largest intensity appears in the first region, at its high-energy end, similarly to $\mathrm{Ne}$ atom [50].

Table IV shows the results of our calculations for the main Auger transition channels in $\mathrm{H}_{2} \mathrm{O}$. We tested two models for the continuum orbital: a plane wave and a Coulomb wave with a uniform effective charge of 4.9 for all channels, which is derived from the Slater rules applied to an isolated oxygen atom. Spectral Auger profiles based on our calculations are shown in the two lower panels of Fig. 4. These profiles were obtained by convolution of the calculated intensities with a Gaussian function with a fixed full width at half maximum equal of $1.0 \mathrm{eV}$. Table IV also includes the transition rates computed by Inhester et al. [56], which are the most accurate in terms of reproducing the experimental profile and can serve as the reference for individual partial widths.

The analysis of Table IV and Fig. 4 shows that our method is able to reproduce the main features of the spectrum, however, some of the channel rates are sig- nificantly over- or under-estimated. The model with the plane wave assigns much too high intensities to triplet channels (depicted with green bars in Fig. 4) between 480 and $475 \mathrm{eV}$. This is rectified when the Coulomb wave is employed to represent the continuum orbital: according to Table IV the rates for triplet channels obtained with the Coulomb wave agree well with the reference theoretical data. A shortcoming, which is not remedied by the Coulomb wave model, is the overestimation of the lowest ${ }^{1} \mathrm{~A}_{1}$ channel and underestimation of the highest ${ }^{1} \mathrm{~A}_{1}$ and ${ }^{1} \mathrm{~B}_{1}$ channels. As in the case of $\mathrm{Ne}$, this problem can be traced back to the interchannel coupling, neglected in our approach. Indeed, the ${ }^{1} \mathrm{~A}_{1}\left(2 a_{1}^{-2}\right)$ channel with two inner vacancies corresponds to the $(2)^{1} \mathrm{~S}$ channel in Ne decay for which the rate was also significantly overestimated, whereas the two channels with the highest energy $\left({ }^{1} \mathrm{D}\right.$ and $\left.(2)^{1} \mathrm{~S}\right)$ turned out to be underestimated. Our results with significantly overestimated the lowest ${ }^{1} \mathrm{~A}_{1}$ channel rate are in line with previous calculations based on Stieltjes imaging or Dyson propagator method [53, 57]. Calculation from [56] have shown also that it is necessary to include nuclear dynamics effects to obtain the correct shape of the peaks at the high-energy end, and that a homogeneous broadening is not sufficient.

As far as the total Auger decay width is concerned, our models predict $175.1 \mathrm{meV}$ for a plane wave and $121.7 \mathrm{meV}$ for a Coulomb wave with $Z_{\text {eff }}=4.9$. These numbers should be compared with the experimentally determined natural linewidth of $K$-shell vacancy in 


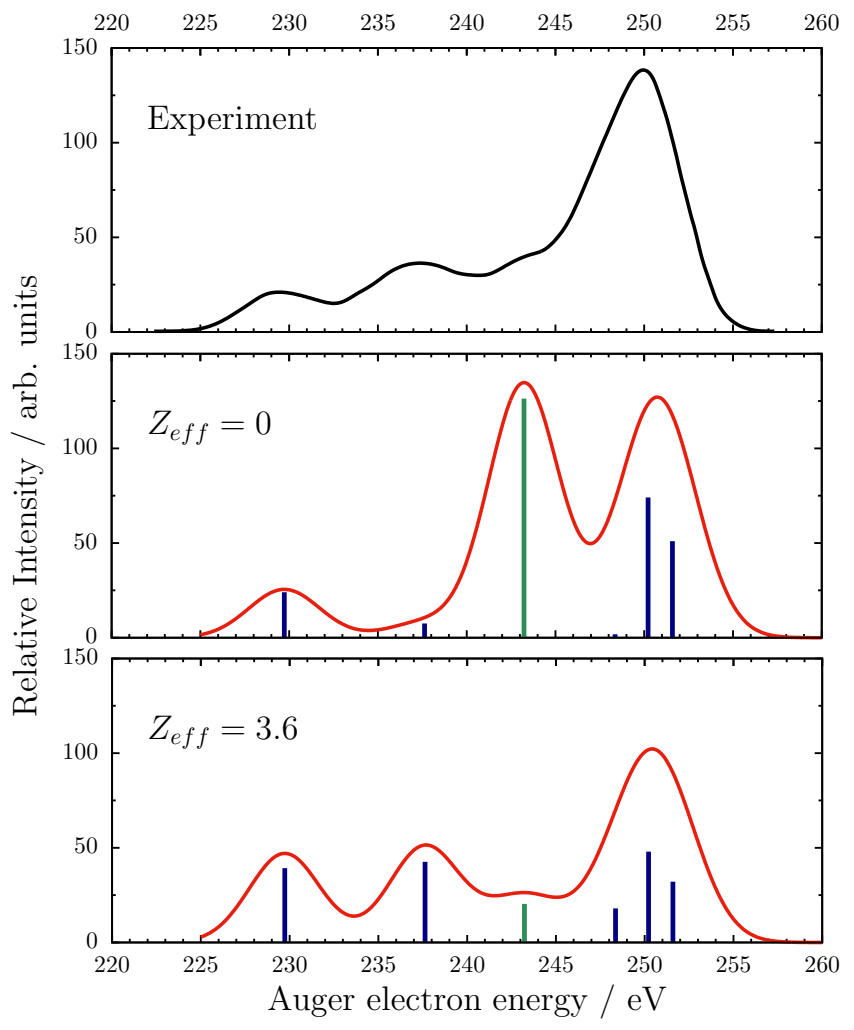

FIG. 5. Experimental [59] and computed Auger spectra for singly ionized methane molecule. Middle and bottom panels show the calculations with a plane wave and a Coulomb wave, respectively. Theoretical curves obtained from the stick spectra by assuming constant Gaussian broadening of 2.0 eV FWHM. Dark-blue sticks correspond to singlet channels while green sticks to triplet channels, respectively.

$\mathrm{H}_{2} \mathrm{O}$ of $160 \pm 5 \mathrm{meV}$ [58]. Thus, as for Ne, the model with a plane wave overestimates the total width, whereas the Coulomb wave underestimates it. The underestimation is expected, because our calculations only account for a single-electron Auger decay pathway, neglecting other possible pathways such as via radiative decay or via coupling to the continua with two or more Auger electrons. Hence, our model with the Coulomb wave provides a lower bound of the total lifetime.

\section{C. $\mathbf{C H}_{4}\left(1 s^{-1}\right)$}

In terms of symmetry considerations, $\mathrm{CH}_{4}$ is an intermediate case between the isoelectronic $\mathrm{Ne}$ atom and $\mathrm{H}_{2} \mathrm{O}$ molecule. It has a ground state configuration of $1 a_{1}^{2} 2 a_{1}^{2} 1 t_{2}^{6}$, with degenerate $t_{2}$ orbitals. From this closed-shell configuration one can derive 7 main channels for the $K-L L$ Auger decay, which is less than for $\mathrm{H}_{2} \mathrm{O}$ (16), but more than for $\mathrm{Ne}$ (5). The Auger electron spectrum for $\mathrm{CH}_{4}$ following single $K$-shell ionization has been measured a few times with different techniques, yielding very consistent shapes [59-61]. The spectrum is simple and features three distinct peaks of decreasing intensities, when moving from high to low electron kinetic energy (see the upper panel of Fig. 5), with peak maxima located around 250.0, 237.5, and $229.6 \mathrm{eV}$. Similarly to $\mathrm{H}_{2} \mathrm{O}$, the three peaks represent transitions to doubly ionized states with holes in either (1) the outer valence shell only, (2) both outer and inner valence shells, or (3) the inner valence shell only, with the first case yielding the largest intensity.

Table $\mathrm{V}$ and Fig. 5 show the results of our calculations of Auger spectra for $\mathrm{CH}_{4}$. The Coulomb wave effective charge of 3.6 is based on the Slater rules applied to an isolated $\mathrm{C}$ atom. Just as with $\mathrm{Ne}$ and $\mathrm{H}_{2} \mathrm{O}$, the main effect of the Coulomb wave is to reduce the intensity in the triplet $T_{2}$ channel, which is considerably overestimated by the plane-wave model. The model with the Coulomb wave reproduces all the main features of the spectrum quite well, however, there is still an imbalance between the intensities for singlet decay channels. Our model also neglects the contributions from the shake-up satellite transitions, which affect the spectrum in the region around 240-250 eV [62].

For the total one-electron $K-L L$ Auger width our models with plane wave and Coulomb wave predicts 95.0 and $66.8 \mathrm{meV}$, respectively. Direct comparison with experimentally determined natural linewidth of $1 a_{1}^{-1}$ core-hole state in $\mathrm{CH}_{4}$ is more difficult as values reported from different measurements vary significantly, ranging from $83 \pm 10 \mathrm{meV}$ to $120 \pm 10 \mathrm{meV}$ [63-66]. Nevertheless, it is clear that the model with the Coulomb wave tends to underestimates the total width, while the model with the plane wave gives more realistic estimation of the total width, however, is it more a consequence of a fortuitous error cancellation.

\section{D. $\mathrm{CO}_{2},\left(\mathrm{C}\right.$-edge $\left.1 s^{-1}\right)$}

$\mathrm{CO}_{2}$ is another small molecule being extensively used for benchmark studies in core-level spectroscopies. It has two distinct $K-L L$ Auger spectra due to ionization from either carbon or oxygen $K$-shell, and both have been reported and scrutinized several times in the literature [50, 67-72]. Here we focus only on carbon $K-L L$ Auger spectrum, as for this case due to symmetry we can apply our models for the continuum electron in a straightforward manner. Outside the $K$-shell the electronic configuration of $\mathrm{CO}_{2}$ in the ground state is the following: $\left(3 \sigma_{g}^{2}\right)\left(2 \sigma_{u}^{2}\right)\left(4 \sigma_{g}^{2}\right)\left(3 \sigma_{u}^{2}\right)\left(1 \pi_{u}^{4}\right)\left(1 \pi_{g}^{4}\right)$. It has a much larger number of possible channels with two hole vacancies in the valence shell than $\mathrm{H}_{2} \mathrm{O}$ or $\mathrm{CH}_{4}$. The experimental Auger spectrum from the carbon $K$-edge, shown in upper panel of Fig. 6, has most of its intensity gathered in the two largest peaks around $250 \mathrm{eV}$. There are also two pronounced sharp peaks at higher kinetic energy (254-258 eV) and a series of much broader 
TABLE V. Energies (in eV), total $\Gamma_{\text {tot }}$ and partial widths $\Gamma_{i}$ (in meV) for the main channels in the Auger decay of $K$-shell ionized methane. Calculations of the widths used either a plane wave or a Coulomb wave with effective charge $Z_{\text {eff }}=3.6$ for all channels.

\begin{tabular}{l|c|c|c}
\hline \hline \multirow{2}{*}{ Channel } & \multirow{2}{*}{ Energy / eV } & \multicolumn{2}{|c}{$\Gamma_{i} / \mathrm{meV}$} \\
\cline { 3 - 4 } & & $Z_{\text {eff }}=0$ & $Z_{\text {eff }}=3.6$ \\
\hline${ }^{3} \mathrm{~T}_{1}$ & 252.27 & 0.1 & 0.0 \\
${ }^{1} \mathrm{E}$ & 251.59 & 17.0 & 10.7 \\
${ }^{1} \mathrm{~T}_{2}$ & 250.22 & 24.7 & 16.0 \\
${ }^{1} \mathrm{~A}_{1}$ & 248.36 & 0.6 & 6.0 \\
${ }^{3} \mathrm{~T}_{2}$ & 243.23 & 42.1 & 6.8 \\
${ }^{1} \mathrm{~T}_{2}$ & 237.63 & 2.5 & 14.2 \\
${ }^{1} \mathrm{~A}_{1}$ & 229.73 & 8.0 & 13.1 \\
\hline$\Gamma_{\text {tot }}$ & & 95.0 & 66.8 \\
\hline \hline
\end{tabular}

TABLE VI. Energies (in eV) and partial widths $\Gamma_{i}$ (in meV) for the main channels in the Auger decay of carbon-edge $K$-shell ionized $\mathrm{CO}_{2}$. Calculations of the widths used either a plane wave or a Coulomb wave with effective charge $Z_{\text {eff }}=3.6$ for all channels.

\begin{tabular}{l|c|c|c}
\hline \hline \multirow{2}{*}{ Channel } & \multirow{2}{*}{ Energy / eV } & \multicolumn{2}{|c}{$\Gamma_{i} / \mathrm{meV}$} \\
\cline { 3 - 4 } & & $Z_{\text {eff }}=0$ & $Z_{\text {eff }}=3.6$ \\
\hline${ }^{1} \Delta_{g}\left(1 \pi_{g}^{-2}\right)$ & 259.23 & 1.0 & 0.7 \\
${ }^{1} \Sigma_{g}^{+}\left(1 \pi_{g}^{-2}\right)$ & 258.68 & 0.3 & 0.2 \\
${ }^{3} \Pi_{u}\left(1 \pi_{g}^{-1} 3 \sigma_{u}^{-1}\right)$ & 256.09 & 2.5 & 0.6 \\
${ }^{1} \Pi_{u}\left(1 \pi_{g}^{-1} 3 \sigma_{u}^{-1}\right)$ & 255.39 & 0.7 & 0.8 \\
${ }^{1} \Pi_{g}\left(1 \pi_{g}^{-1} 4 \sigma_{g}^{-1}, 1 \pi_{u}^{-1} 3 \sigma_{u}^{-1}\right)$ & 254.84 & 2.2 & 1.6 \\
${ }^{1} \Sigma_{g}^{+}\left(3 \sigma_{u}^{-2}, 4 \sigma_{g}^{-2}\right)$ & 252.18 & 1.1 & 3.1 \\
${ }^{3} \Sigma_{u}^{+}\left(4 \sigma_{g}^{-1} 3 \sigma_{u}^{-1}\right)$ & 252.01 & 3.3 & 0.7 \\
${ }^{1} \Delta_{g}\left(1 \pi_{u}^{-2}\right)$ & 250.08 & 10.0 & 6.3 \\
${ }^{3} \Pi_{u}\left(4 \sigma_{g}^{-1} 1 \pi_{u}^{-1}\right)$ & 249.75 & 8.1 & 1.6 \\
${ }^{1} \Sigma_{g}^{+}\left(1 \pi_{u}^{-2}\right)$ & 249.28 & 2.4 & 3.0 \\
${ }^{1} \Pi_{g}\left(3 \sigma_{u}^{-1} 1 \pi_{u}^{-1}, 4 \sigma_{g}^{-1} 1 \pi_{g}^{-1}\right)$ & 248.41 & 4.5 & 2.9 \\
${ }^{1} \Pi_{u}\left(4 \sigma_{g}^{-1} 1 \pi_{u}^{-1}, 3 \sigma_{u}^{-1} 1 \pi_{g}^{-1}\right)$ & 247.67 & 0.4 & 2.7 \\
${ }^{1} \Sigma_{u}^{+}\left(4 \sigma_{g}^{-1} 3 \sigma_{u}^{-1}\right)$ & 243.40 & 0.1 & 1.4 \\
${ }^{1} \Sigma_{g}^{+}\left(4 \sigma_{g}^{-2}, 3 \sigma_{u}^{-2}\right)$ & 243.02 & 3.0 & 1.8 \\
${ }^{3} \Pi_{u}\left(2 \sigma_{u}^{-1} 1 \pi_{g}^{-1}\right)$ & 238.49 & 3.8 & 0.9 \\
${ }^{1} \Pi_{u}\left(2 \sigma_{u}^{-1} 1 \pi_{g}^{-1}\right)$ & 237.02 & 0.7 & 1.7 \\
${ }^{3} \Sigma_{u}^{+}\left(3 \sigma_{g}^{-1} 3 \sigma_{u}^{-1}, 4 \sigma_{g}^{-1} 2 \sigma_{u}^{-1}\right)$ & 234.67 & 5.7 & 1.0 \\
${ }^{1} \Sigma_{g}^{+}\left(2 \sigma_{u}^{-1} 3 \sigma_{u}^{-1}, 3 \sigma_{g}^{-1} 4 \sigma_{g}^{-1}\right)$ & 234.17 & 2.3 & 6.4 \\
${ }^{3} \Pi_{u}\left(3 \sigma_{g}^{-1} 1 \pi_{u}^{-1}\right)$ & 230.72 & 7.6 & 1.5 \\
${ }^{1} \Pi_{g}\left(2 \sigma_{u}^{-1} 1 \pi_{u}^{-1}, 3 \sigma_{g}^{-1} 1 \pi_{g}^{-1}\right)$ & 226.77 & 3.4 & 2.2 \\
\hline \hline
\end{tabular}

peaks at lower energies, from 245 to $225 \mathrm{eV}$. Table VI and Fig. 6 show the computed Auger spectra. In the calculations with the Coulomb wave, we assumed the effective charge of 3.6 (same as in $\mathrm{CH}_{4}$ ), centered on the carbon atom. As one can see both our models reproduce the experimental spectrum quite well, and the predicted relative intensities and positions of the peaks allow for unique assignment of the observed features in the spectrum. Interestingly, even with the simplest model of the plane wave, the overall shape of the spectrum is as good as in the model with the Coulomb wave. However, the analysis of Table VI clearly shows that the plane wave orbital largely overestimates the contributions from the triplet channels, the behavior observed in other studied molecules. At the same time, the overestimated triplet channels are located energetically relatively close to the singlet channels which are truly populated in the Auger decay. Therefore, even though the exact assignment of the peaks based on the calculations with the plane wave can be incorrect, the total shape of spectrum might turn out surprisingly well, in particular for larger molecules with many accessible final states contributing 


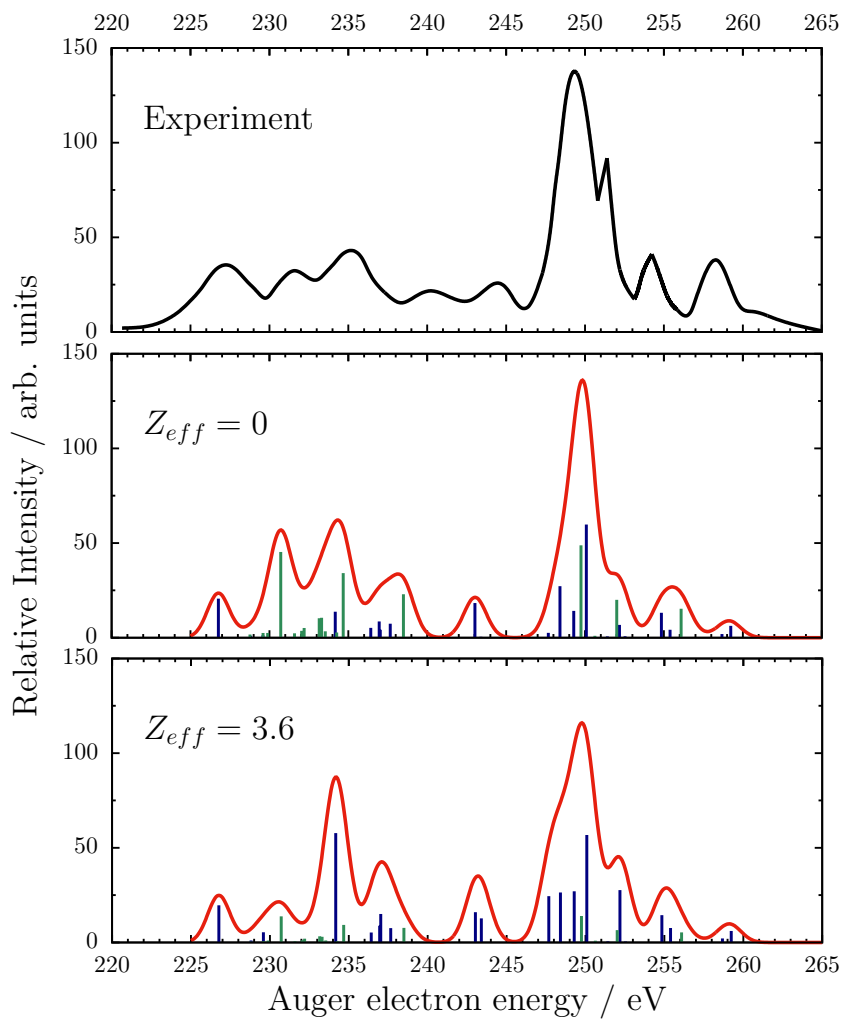

FIG. 6. Experimental [67] and computed Auger spectra for carbon $\mathrm{K}$-edge in $\mathrm{CO}_{2}$. Middle and bottom panels show the calculations with a plane wave and a Coulomb wave, respectively. Theoretical curves obtained from the stick spectra by assuming constant Gaussian broadening of $0.7 \mathrm{eV}$ FWHM. Dark-blue sticks correspond to singlet channels while green sticks to triplet channels, respectively.

to the observed signal. As shown above, also for $\mathrm{CO}_{2}$, the Coulomb wave reverses the Auger transition intensities from triplet to singlet channels. Interestingly, the Coulomb wave performs also quite well in case of channels with highest kinetic energy dominated by two vacancies in the highest occupied molecular orbital, $1 \pi_{g}$, which, for symmetry reason, does not contain carbon character. Overall, the Coulomb wave allows the interpretation of the main features in the $\mathrm{CO}_{2}$ Auger spectrum, which agrees very well with previously published results based on $\operatorname{ADC}(2)$ calculations and the atomic two-hole population analysis $[67,68,70]$. The main discrepancy from the measured spectrum occurs around the region with two relatively broad peaks centered at around 244 and $240 \mathrm{eV}$. Our EOM-DIP calculations predict in this region two almost degenerate channels $\left({ }^{1} \Sigma_{u}^{+}\right.$ and ${ }^{1} \Sigma_{g}^{+}$), with energy difference of only $0.4 \mathrm{eV}$ and centered at $243 \mathrm{eV}$. One important aspect, which is neglected in our study, is nuclear dynamics and broadening of the Auger spectrum due to the vibrational structure in the initial and final states. Theoretical spectra depicted in Fig. 6 assumed a fixed Gaussian profile with the FWHM of $0.7 \mathrm{eV}$. This is a crude approximation, given the fact that the fits to the experimental spectrum yielded the widths of the peaks varying from $0.3 \mathrm{eV}$ up to a few eV [67]. The effect of the nuclear dynamics contributes also to the shift of the peaks relative to the vertical Auger transitions, assumed in our work.

\section{E. $\mathrm{Ne}\left(1 s^{-1} 3 p\right)$}

In the resonant Auger process the initial state for auto-ionization is created by a resonant excitation of a core electron to a vacant valence orbital (see Fig. 1). Due to the coupling between the excited electron and the remaining core, there is a significant increase in the number of possible decay channels, compared to regular Auger process. Additionally, more diffuse character of the initial core-excited state makes shake-up and shake-down processes more pronounced. Therefore, the resonant Auger spectra have more complicated pattern of peaks and pose greater challenge for theoretical interpretation. As a first example here, we consider the Auger decay following the $1 s \rightarrow 3 p\left({ }^{1} P_{1}\right)$ excitation in $\mathrm{Ne}$ atom. The relaxation of the $1 s^{-1} 3 p^{1}{ }^{1} P_{1}$ state proceeds via multiple autoionization (Auger cascade), with one Auger electron ejected after another [75-77]. Here we consider only the first step of this process in which singly charged $\mathrm{Ne}^{+}$ions are formed. The top panel in Fig. 7 shows the experimental Auger emission spectrum of $1 s^{-1} 3 p^{1} \mathrm{Ne}$, covering the energy range from 800 to $820 \mathrm{eV}$, where most of the intensities are located. There are three main classes of Auger processes occurring in the decay of $1 s^{-1} 3 p^{1}$ Ne: $(a)$ spectator processes of the type $1 s 2 s^{2} 2 p^{6} 3 p \rightarrow 1 s^{2}(2 s 2 p)^{6} 3 p+e^{-}$, where the initially excited electron does not participate in the autoionization, (b) shake-up processes of the type $1 s 2 s^{2} 2 p^{6} 3 p \rightarrow$ $1 s^{2}(2 s 2 p)^{6} 4 p+e^{-}$where there is additional excitation to the higher Rydberg level, and (c) participator processes of the type $1 s 2 s^{2} 2 p^{6} 3 p \rightarrow 1 s^{2}(2 s 2 p)^{7}+e^{-}$, where the electron from the Rydberg orbital participates directly in the autoionization. From the previous studies it is well known that the decay of $1 s^{-1} 3 p^{1}$ state is dominated by the spectator processes [76-78]. The highest peaks in Fig. 7 can be assigned to channels with $\mathrm{Ne}^{+}$ valence configurations of $2 s^{2} 2 p^{4}\left({ }^{1} D\right) 3 p$. There are also significant contributions from shake-up transitions with the final valence configurations of $2 s^{2} 2 p^{4}\left({ }^{1} D\right) 4 p$, while participator processes are the least important.

Table VII shows the results of our calculations, compared with available experimental data. As far as channel energies are considered, our computational model gives good predictions only for participator channels, but performs rather poorly for spectator or shake-up transitions, which turn out to be blue-shifted by around $10 \mathrm{eV}$. As the initial excitation energy for $1 s \rightarrow 3 p$ transition is well reproduced in our model $(866.86 \mathrm{eV}$ vs. experimental value $867.13 \mathrm{eV}$ ), the underestimated chan- 
TABLE VII. Energies (in eV), partial widths $\Gamma_{i}$ (in $\mathrm{meV}$ ), and relative intensities $I_{i}$ for the Auger decay of $1 s \rightarrow 3 p$ coreexcited Ne atom from present calculations, compared with experimental data. Calculations of the widths used either a plane wave or a Coulomb wave with effective charge $Z_{\text {eff }}=5.85$ for $1 h$-type channels and $Z_{\text {eff }}=6.2$ for $2 h 1 p$-type channels. Relative intensities $I_{i}$ are given in $\%$ with respect to the intensity of the dominant ${ }^{2} F$ channel.

\begin{tabular}{|c|c|c|c|c|c|c|c|}
\hline \multirow{2}{*}{ Channel } & \multicolumn{2}{|c|}{ Energy / eV } & \multicolumn{2}{|c|}{$\Gamma_{i} / \mathrm{meV}$} & \multicolumn{3}{|c|}{$I_{i}$} \\
\hline & Present & Exp. [73] & $Z_{e f f}=0$ & $Z_{e f f}=5.85 / 6.2$ & $Z_{e f f}=0$ & $Z_{e f f}=5.85 / 6.2$ & Exp. [74] \\
\hline${ }^{2} P\left(2 s^{2} 2 p^{5}\right)$ & 845.52 & 845.47 & 1.0 & 0.7 & 2.3 & 2.4 & \\
\hline${ }^{2} S\left(2 s^{1} 2 p^{6}\right)$ & 818.39 & 818.56 & 1.0 & 0.02 & 2 & 0.1 & \\
\hline${ }^{2} P\left(2 s^{2} 2 p^{4}\left({ }^{3} P\right) 3 p^{1}\right)$ & 803.65 & 814.04 & 6.6 & 4.5 & 15 & 15 & 3 \\
\hline${ }^{2} F\left(2 s^{2} 2 p^{4}\left({ }^{1} D\right) 3 p^{1}\right)$ & 801.46 & 811.54 & 43.5 & 29.2 & 100 & 100 & 100 \\
\hline${ }^{2} D\left(2 s^{2} 2 p^{4}\left({ }^{1} D\right) 3 p^{1}\right)$ & 800.66 & 811.18 & 33.3 & 22.4 & 76 & 76 & 73 \\
\hline${ }^{2} P\left(2 s^{2} 2 p^{4}\left({ }^{1} D\right) 3 p^{1}\right)$ & 799.33 & 811.28 & 10.7 & 8.6 & 25 & 29 & 37 \\
\hline${ }^{2} P\left(2 s^{2} 2 p^{4}\left({ }^{3} P\right) 4 p^{1}\right)$ & 798.64 & 809.07 & 3.1 & 5.3 & 7 & 18 & \\
\hline${ }^{2} P\left(2 s^{2} 2 p^{4}\left({ }^{1} S\right) 3 p^{1}\right)$ & 796.21 & 807.70 & 9.5 & 6.8 & 22 & 23 & 32 \\
\hline${ }^{2} F\left(2 s^{2} 2 p^{4}\left({ }^{1} D\right) 4 p^{1}\right)$ & 794.75 & 806.28 & 29.2 & 19.7 & 67 & 67 & \\
\hline${ }^{2} D\left(2 s^{2} 2 p^{4}\left({ }^{1} D\right) 4 p^{1}\right)$ & 794.57 & 806.17 & 18.2 & 12.3 & 42 & 42 & \\
\hline${ }^{2} P\left(2 s^{2} 2 p^{4}\left({ }^{1} D\right) 4 p^{1}\right)$ & 794.21 & 806.16 & 5.0 & 3.4 & 12 & 12 & \\
\hline${ }^{2} P\left(2 s^{2} 2 p^{4}\left({ }^{1} S\right) 4 p^{1}\right)$ & 792.57 & 802.52 & 2.0 & 4.0 & 4 & 14 & \\
\hline
\end{tabular}

nel energies are consequence of a poor description of $\mathrm{Ne}^{+}$ excited Rydberg states. When the closed-shell Ne atom serves as a reference state for EOM-CC calculations, $\mathrm{Ne}^{+}$excited states dominated by configurations such as $1 s^{2}(2 s 2 p)^{6} 3 p$ or $1 s^{2}(2 s 2 p)^{6} 4 p$ can only be obtained by means of $R_{2}$ operator (or higher order). Therefore, due to the lack of triple excitations in the EOM-CCSD model, these states are described less accurately than the states of $1 h$ character. The resulting insufficient description of correlation and relaxation effects results in $\sim 10 \mathrm{eV}$ errors.

As for previous cases, we have computed Auger partial widths with either a plane wave or Coulomb wave to model the continuum electron. For the Coulomb wave the chosen effective charge $Z=5.85$ for participator channels and $Z=6.2$ for spectator channels (it is assumed that screening from the spectator electron is negligible). To facilitate the comparison of the computed Auger intensities with the experimental data, the theoretical spectra shown in Fig. 7 were obtained in such a way that all peaks due to the channels with an occupied Rydberg level have been shifted by $10.1 \mathrm{eV}$ in order to match the experimental position of the most intense peak at $811.5 \mathrm{eV}$, which is due to the ${ }^{2} F\left(2 s^{2} 2 p^{4}\left({ }^{1} D\right) 3 p^{1}\right)$ channel. Additionally, theoretical spectra have been normalized to the height of the dominant peak, and a constant broadening of $0.1 \mathrm{eV}$ with a Gaussian envelope have been introduced. The agreement between the calculated and experimental spectra is rather qualitative. Our calculations correctly describe the relative intensities of the most pronounced transitions, however, there is a mismatch in the positions and intensities of the satellite peaks. What is striking is that the relative intensities obtained with the plane wave and the Coulomb wave are almost identical. This can be

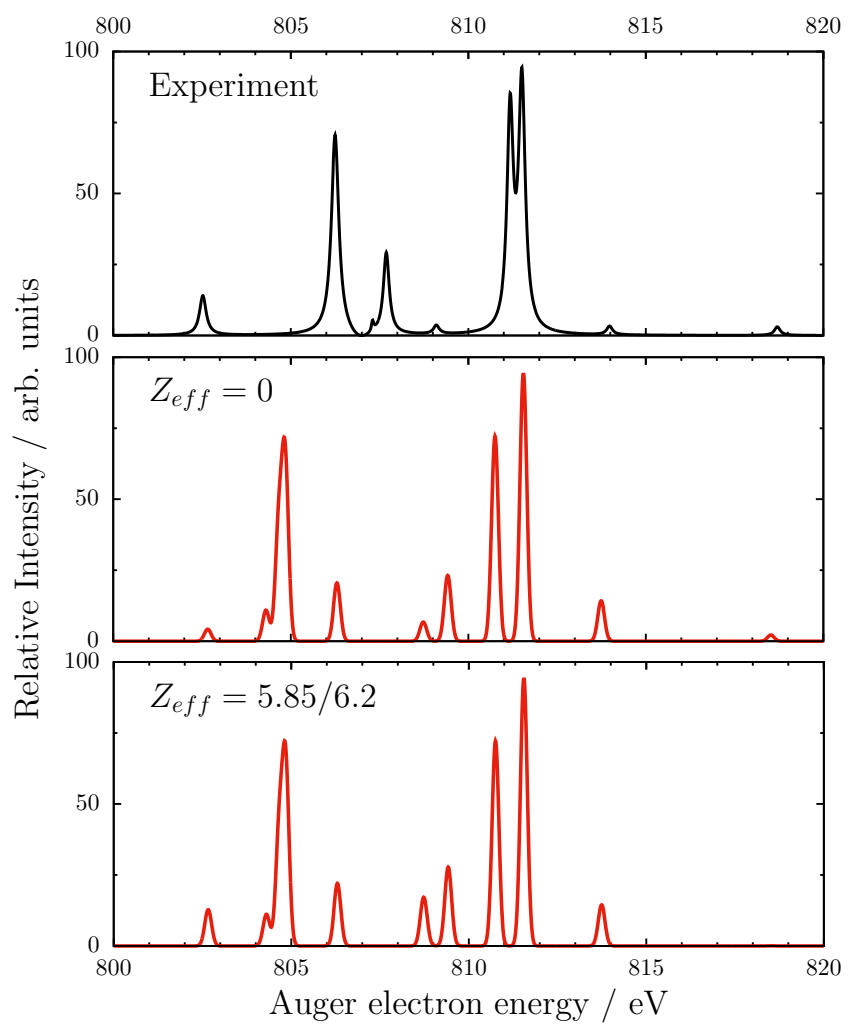

FIG. 7. Experimental [74] and computed resonant Auger spectra following $1 s \rightarrow 3 p$ transition in Ne atom. Middle and bottom panels show calculations with a plane wave and a Coulomb wave, respectively. Theoretical curves were obtained from the stick spectra by assuming constant Gaussian broadening of $0.1 \mathrm{eV} \mathrm{FWHM}$ and normalized to the height of the dominant ${ }^{2} F$ channel. All theoretical peaks corresponding to spectator-type transitions were shifted by $10.1 \mathrm{eV}$ to match the position of the experimental ${ }^{2} F$ channel. 
explained by the fact that vast majority of the decay channels have $2 s^{2} 2 p^{4}\left({ }^{1} D\right) 3 p / 4 p$ configurations, which are associated with the emission of $d$-wave electron. As we have seen in the case of normal Auger decay in $\mathrm{Ne}$, the plane wave performs exceptionally well for $2 s^{2} 2 p^{4}\left({ }^{1} D\right)$ channel ( $d$-wave emission), and the same behavior can be expected for the resonant Auger effect. As was shown in the Ref.[79], due to a local nature of the Auger process, the higher angular momentum of the emitted electron is, the less important the potential from the remaining core state is. This observation is confirmed by our calculations: for the $d$-wave Auger electrons the plane wave performs as good as the Coulomb wave. For most channels shown in Table VII, introducing a Coulomb wave merely results in the overall reduction of the Auger intensities, while the relative intensities remain almost unaffected. Similar behavior have been observed for these transitions in recent $a b$ initio calculations based on the RASSCF wave function [79].

\section{F. $\mathbf{C O}_{2}\left(\right.$ C-edge, $\left.1 s \rightarrow \pi^{*}\right)$}

To assess the performance of our model for molecular resonant Auger decay, we have chosen C-edge coreexcited $1 s \rightarrow \pi_{u}^{*}\left({ }^{1} \Pi_{u}\right)$ transition in $\mathrm{CO}_{2}$ as a test case. This state has been throughly investigated, and proved to be challenging for precise computational modeling, due to both geometrical effects and complex pattern of possible decay channels [80-83]. In particular, the Renner-Teller effect in the core-excited ${ }^{1} \Pi_{u}$ state makes the Auger spectra highly sensitive to the exact excitation energy [84]. After the degeneracy of ${ }^{1} \Pi_{u}$ state is lifted for bent geometry, two overlapping resonant states appear, and non-adiabatic quantum-dynamical calculations is required to properly account for possible vibrational and electronic interferences.

Measured resonant electron Auger spectrum following $\mathrm{C} 1 s \rightarrow \pi_{u}^{*}\left({ }^{1} \Pi_{u}\right)$ excitation in $\mathrm{CO}_{2}$ is shown in the uppermost panel of Fig. 8. Previous theoretical studies utilizing comparisons with valence photoionization and normal Auger spectra, allowed to interpret satisfactorily the resonant Auger $\mathrm{CO}_{2}$. The region above electron kinetic energy of $270 \mathrm{eV}$ (binding energy less than $20 \mathrm{eV}$ ) is exclusively due to participator transitions to $\mathrm{CO}_{2}^{+}$states with one hole in the orbital from outer valence shell $\left(1 \pi_{g}^{-1}, 1 \pi_{u}^{-1}, 3 \sigma_{u}^{-1}\right.$, or $\left.4 \sigma_{g}^{-1}\right)$. Additional contributions from participator-like transitions to states with single hole in the inner valence shell $\left(2 \sigma_{u}^{-1}\right.$ or $3 \sigma_{g}^{-1}$ ) are located between 250 to $255 \mathrm{eV}$ (with binding energy 35 to $40 \mathrm{eV}$ ). The rest of the spectrum, including all the peaks with electron kinetic energy between 255 and $270 \mathrm{eV}$, can be assigned to the spectator decay, where the final $\mathrm{CO}_{2}^{+}$states have dominant configurations with two holes in the valence shell and the excited electron in the $\pi_{u}^{*}$ orbital.

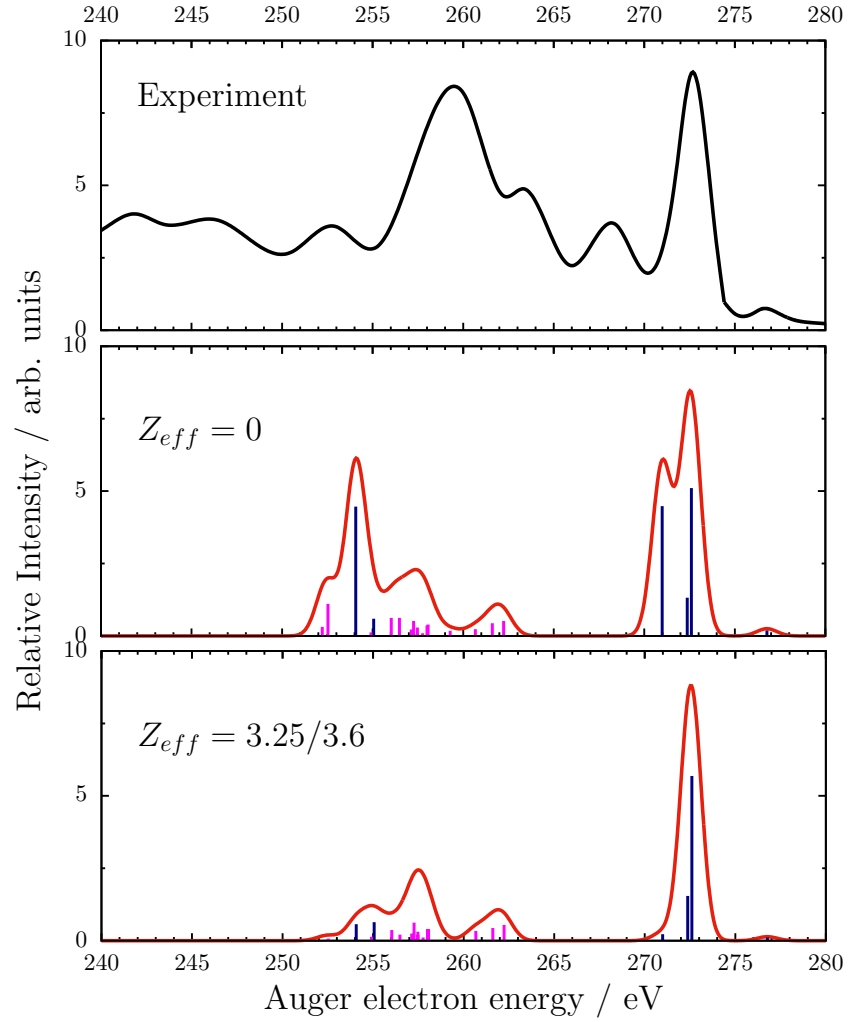

FIG. 8. Experimental [80] and computed resonant Auger spectra following carbon-edge $1 s \rightarrow \pi_{u}^{*}$ excitation in $\mathrm{CO}_{2}$. Middle and bottom panels show the calculations with a plane wave and a Coulomb wave, respectively. Theoretical curves were obtained from the stick spectra by assuming constant Gaussian broadening of 0.7 eV FWHM. Dark-blue sticks represents participator channels while magenta sticks are spectator channels.

Table VIII and Fig. 8 show the results of our calculations. Similarly to the resonant Auger spectrum in Ne, for the model with the Coulomb wave we applied two different effective charges: $Z_{\text {eff }}=3.25$ for participator channels and $Z_{\text {eff }}=3.6$ for spectator channels, which are derived from Slater rules applied to isolated $\mathrm{C}$ atom with the assumption that the electron from the outer $\pi_{u}^{*}$ orbital does not contribute to the screening. Our theoretical spectra reproduce only partly the experimental features. Certainly, our predictions are more accurate for participator channels than for spectator channels. Our models correctly identify the highest peak due to $\left(\pi_{u}^{-1}\right)^{2} \Pi_{u}$ state. Also the relative intensities and positions of other participator channels are reproduced reasonably well. The model with the Coulomb wave does a better job, as the plane wave overestimates the intensities of the two ${ }^{2} \Sigma_{g}^{+}$participator states. Our calculations fail with regard to the spectator channels. In our model the onset of spectator channels appear at around $262 \mathrm{eV}$ (binding energy $28 \mathrm{eV}$ ), while in the measured spectrum it is at around $268 \mathrm{eV}$ (binding energy $22 \mathrm{eV}$ ). As in 
TABLE VIII. Energies (in eV) and partial widths $\Gamma_{i}$ (in meV) for the main channels in the Auger decay of $1 s \rightarrow \pi^{*} \mathrm{C}$-edge core-excited $\mathrm{CO}_{2}$ from present calculations. Calculations of the widths used either a plane wave or a Coulomb wave with effective charge $Z_{\text {eff }}=3.25$ for $1 h$-type channels and $Z_{e f f}=3.6$ for $2 h 1 p$-type channels.

\begin{tabular}{|c|c|c|c|}
\hline \multirow{2}{*}{ Channel } & \multirow{2}{*}{ Energy / eV } & \multicolumn{2}{|c|}{$\Gamma_{i} / \mathrm{meV}$} \\
\hline & & $Z_{e f f}=0$ & $Z_{e f f}=3.25 / 3.6$ \\
\hline${ }^{2} \Pi_{g}\left(1 \pi_{g}^{-1}\right)$ & 276.77 & 0.4 & 0.14 \\
\hline${ }^{2} \Pi_{u}\left(1 \pi_{u}^{-1}\right)$ & 272.60 & 10.2 & 7.1 \\
\hline${ }^{2} \Sigma_{u}^{+}\left(3 \sigma_{u}^{-1}\right)$ & 272.37 & 2.6 & 1.9 \\
\hline${ }^{2} \Sigma_{g}^{+}\left(4 \sigma_{g}^{-1}\right)$ & 270.99 & 9.0 & 0.3 \\
\hline${ }^{2} \Sigma_{u}^{+}\left(2 \sigma_{u}^{-1}\right)$ & 255.06 & 1.2 & 0.8 \\
\hline${ }^{2} \Sigma_{g}^{+}\left(3 \sigma_{g}^{-1}\right)$ & 254.07 & 8.9 & 0.7 \\
\hline${ }^{2} \Pi_{u}\left(1 \pi_{g}^{-2} \pi_{u}^{*}, 1 \pi_{u}^{-2} \pi_{u}^{*}\right)$ & 262.23 & 1.0 & 0.7 \\
\hline${ }^{2} \Pi_{u}\left(1 \pi_{g}^{-2} \pi_{u}^{*}, 1 \pi_{u}^{-2} \pi_{u}^{*}\right)$ & 261.61 & 0.9 & 0.5 \\
\hline${ }^{2} \Pi_{u}\left(1 \pi_{u}^{-2} \pi_{u}^{*}, 1 \pi_{u}^{-2} \pi_{u}^{*}\right)$ & 260.67 & 0.5 & 0.4 \\
\hline${ }^{2} \Sigma_{g}^{+}\left(1 \pi_{g}^{-2} \sigma_{g}^{*}\right)$ & 259.28 & 0.4 & 0.02 \\
\hline${ }^{2} \Sigma_{g}^{+}\left(1 \pi_{g}^{-1} 3 \sigma_{u}^{-1} \pi_{u}^{*}\right)$ & 258.06 & 0.8 & 0.5 \\
\hline${ }^{2} \Sigma_{u}^{+}\left(1 \pi_{g}^{-1} 4 \sigma_{g}^{-1} \pi_{u}^{*}, 1 \pi_{u}^{-1} 3 \sigma_{u}^{-1} \pi_{u}^{*}\right)$ & 258.00 & 0.7 & 0.5 \\
\hline${ }^{2} \Sigma_{u}^{-}\left(1 \pi_{u}^{-1} 1 \pi_{g}^{-1} \sigma_{g}^{*}\right)$ & 257.76 & 0.2 & 0.1 \\
\hline${ }^{2} \Sigma_{u}^{+}\left(1 \pi_{u}^{-1} 1 \pi_{g}^{-1} \sigma_{g}^{*}\right)$ & 257.47 & 0.6 & 0.4 \\
\hline${ }^{2} \Sigma_{g}^{-}\left(1 \pi_{g}^{-1} 3 \sigma_{u}^{-1} \pi_{u}^{*}\right)$ & 257.47 & 0.1 & 0.2 \\
\hline${ }^{2} \Sigma_{g}^{-}\left(1 \pi_{g}^{-1} 3 \sigma_{u}^{-1} \pi_{u}^{*}\right)$ & 257.34 & 0.1 & 0.1 \\
\hline${ }^{2} \Pi_{u}\left(1 \pi_{g}^{-2} \pi_{u}^{*}, 1 \pi_{g}^{-1} 3 \sigma_{u}^{-1} \sigma_{g}^{*}\right)$ & 257.26 & 1.0 & 0.8 \\
\hline${ }^{2} \Sigma_{u}^{-}\left(1 \pi_{g}^{-1} 4 \sigma_{g}^{-1} \pi_{u}^{*}\right)$ & 257.13 & 0.4 & 0.3 \\
\hline${ }^{2} \Sigma_{g}^{-}\left(1 \pi_{u}^{-1} 4 \sigma_{g}^{-1} \pi_{u}^{*}\right)$ & 256.48 & 1.2 & 0.3 \\
\hline${ }^{2} \Sigma_{g}^{-}\left(1 \pi_{u}^{-1} 4 \sigma_{g}^{-1} \pi_{u}^{*}\right)$ & 256.03 & 1.2 & 0.5 \\
\hline${ }^{2} \Pi_{u}\left(1 \pi_{g}^{-1} 3 \sigma_{u}^{-1} \sigma_{g}^{*}, 1 \pi_{u}^{-1} 4 \sigma_{g}^{-1} \sigma_{g}^{*}\right)$ & 254.91 & 0.2 & 0.2 \\
\hline${ }^{2} \Sigma_{g}^{+}\left(1 \pi_{g}^{-2} \sigma_{g}^{*}, 1 \pi_{g}^{-1} 3 \sigma_{u}^{-1} \pi_{u}^{*}\right)$ & 252.53 & 2.2 & 0.1 \\
\hline${ }^{2} \Sigma_{u}^{+}\left(1 \pi_{g}^{-1} 4 \sigma_{g}^{-1} \pi_{u}^{*}, 1 \pi_{u}^{-1} 3 \sigma_{u}^{-1} \pi_{u}^{*}\right)$ & 252.50 & 0.1 & 0.1 \\
\hline${ }^{2} \Sigma_{g}^{+}\left(1 \pi_{g}^{-1} 3 \sigma_{u}^{-1} \pi_{u}^{*}\right)$ & 252.22 & 0.6 & 0.03 \\
\hline
\end{tabular}

the Ne case, this error can be traced back to the limited ability of the EOM-IP-CCSD ansatz to describe excited states of the $2 h 1 p$ type. Apart from the mismatch in the position, the relative intensities of the spectator channels are also not well reproduced. What one expects for spectator channels is a pattern that closely resembles the regular Auger spectrum (Fig. 6) but is shifted to higher kinetic energy by around $9.7 \mathrm{eV}$. This pattern is not well reproduced, however, neither by the plane wave nor the Coulomb wave model, with both models possibly underestimating significantly the Auger transitions from spectator states.

When comparing the spectra obtained with the plane wave and the Coulomb wave, we can see that the main difference appear for the ${ }^{2} \Sigma_{g}^{+}$channels, both of the participator and spectator types, which are reduced by roughly one order of magnitude when employing the Coulomb wave. Coulomb wave likely provides an improvement over the plane wave as it is known from vibrationally resolved Auger spectra in the participator region that the intensities of the ${ }^{2} \Sigma_{g}^{+}$channels are much lower than for the ${ }^{2} \Pi_{u}$ channel [85]. At the same time, employing the Coulomb wave does not seem to affect most spectator channels and their overall contribution to the Auger spectra. This can be rationalized by the fact that in the region with high density of final states with strongly mixed configurations, details due to the exact shape of the continuum orbital are averaged out. Also, this behavior for spectator channels is consistent with the results for the regular Auger spectrum (Fig. 6) where the plane wave and the Coulomb wave performed almost in the same way. 


\section{CONCLUSIONS AND OUTLOOK}

We have presented numeric results illustrating the performance of the extension of the EOM-CCSD formalism[26] to the computation of Auger decay rates in atoms and molecules. We calculated Auger decay rates using the Feshbach-Fano formalism, with manyelectron EOM-CC states and a continuum orbital describing the outgoing electron approximated by either a plane wave or a Coulomb wave. Our benchmark calculations for the $\mathrm{Ne}$ atom and $\mathrm{H}_{2} \mathrm{O}, \mathrm{CH}_{4}$, and $\mathrm{CO}_{2}$ molecules show that, despite their simplicity, these models for the continuum orbital are able to treat the autoionization of core-exited and core-ionized states reliably, and can be used to explain experimental spectra. The variant with the plane wave is particularly easy to apply, as it is parameter-free and affords fast calculations, even with sizeable orbital basis set. This model can be considered as an extension of statistical approaches[? ? ] that partially accounts for the scattering character of the Auger decay and for the symmetry of the initial and final states. It also provides lowestorder estimation of partial and total transition rates. When applying more-sophisticated models for the continuum orbitals, calculations with pure plane wave can be used for fast, initial screening of decay channels in order to identify those that are important for the given system. Further, due to the localized nature of the Auger process, the higher partial waves contributing to the decay are, the more accurate the estimation based on the plane-wave approximation are expected to be.

Plane-wave description of the continuum orbital has its limitations, and our calculations have shown that this model fails to describe branching ratios between singlet and triplet channels resulting from the same orbital occupations. This deficiency is rectified by using a Coulomb wave instead of a plane wave. Application of the Coulomb wave requires specification of its effective charge and the origin. The effective charge can be chosen based on different criteria; here we have mostly tested the effective charges resulting from Slater rules applied to an isolated atom bearing the core vacancy. In this way, our Coulomb wave model is closely related to the commonly used one-center approximation with pure atomic continuum functions. In general, we have seen that application of the Coulomb wave, even with a simple choice of the effective charge based on Slater rules, improves the partial decay widths, in particular for the normal Auger decay in cases when both singlet and triplet channels are possible. At the same time, the results for $\mathrm{CO}_{2}$ do not differ significantly when moving from the plane-wave to Coulomb-wave model. This is mainly because of the larger density of pos- sible decay channels than in smaller systems like $\mathrm{Ne}$ or $\mathrm{H}_{2} \mathrm{O}$, and the overall effect of averaging when there are multiple closely spaced channels contributing to the spectrum. Therefore, we anticipate that in more complex molecules, the differences between plane wave and Coulomb wave will be reduced. This observation might be helpful in investigations, for example, of the environment effects on the Auger lifetime.

Certainly, the present work does not discuss the full complexity of the problem, and there are important issues that remain to be addressed to improve the predictive power of the methodology. First, a more realistic description of the continuum orbital should be obtained by solving the mean-field problem. A natural basis set to calculate the continuum orbital seems to consist of products of Gaussian and plane wave functions, which would keep the calculations of all integrals fully analytic. Second, the importance of the inter-channel coupling should be explored, and some way to estimate its contribution in molecules should be proposed. Third, to improve results for resonant Auger decay, it is necessary to include the triple excitation operator in the EOM$\mathrm{CC}$ ansatz. Full EOM-CCSDT calculations might be limited to rather small systems; however, approximate models [86, 87] might be sufficient to reduce the error in the description of the satellite and spectator channels. And, finally, the complete treatment of Auger electron spectra in molecules should include the effect of Franck-Condon broadening and vibrational dynamics, which are neglected in the present work.

\section{ACKNOWLEDGMENTS}

This work was supported by the U.S. National Science Foundation (No. CHE-1856342). We thank Dr. Evgeny Epifanovsky from Q-Chem, Inc for his help and guidance in the implementation of mixed gaussian-plane wave integrals.

\section{CONFLICTS OF INTEREST}

A.I.K. is the president and a part-owner of Q-Chem, Inc.

\section{DATA AVAILABILITY STATEMENT}

The data that supports the findings of this study are available within the article and its supplementary material.
[1] J. A. van Bokhoven and C. Lamberti, editors, X-Ray Absorption and X-ray Emission Spectroscopy; Theory and Applications. Wiley \& Sons, Chichester, Germany, 
2016.

[2] S. Mobilio, F. Boscherini, and C. Meneghini, editors, Synchrotron Radiation: Basics, Methods and Applications. Springer, Berlin (Germany), 2014.

[3] U. Bergmann, V. K. Yachandra, and J. Yano, editors, $X$-Ray Free Electron Lasers: Applications in Materials, Chemistry and Biology, Number 18 in Energy and Environment Series. Royal Society of Chemistry, Croydon, U.K., 2017.

[4] M. Nisoli, P. Decleva, F. Calegari, A. Palacios, and F. Martín, Attosecond electron dynamics in molecules, Chem. Rev. 117, 10760 (2017).

[5] P. Norman and A. Dreuw, Simulating X-ray spectroscopies and calculating core-excited states of molecules, Chem. Rev. 118, 7208 (2018).

[6] O. Kostko, B. Bandyopadhyay, and M. Ahmed, Vacuum ultraviolet photoionization of complex chemical systems, Annu. Rev. Phys. Chem. 67, 19 (2016).

[7] L. S. Cederbaum, W. Domcke, and J. Schirmer, Manybody theory of core holes, Phys. Rev. A 22, 206 (1980).

[8] A. I. Krylov, Equation-of-motion coupled-cluster methods for open-shell and electronically excited species: The hitchhiker's guide to Fock space, Annu. Rev. Phys. Chem. 59, 433 (2008).

[9] J. F. Stanton and R. J. Bartlett, The equation of motion coupled-cluster method. A systematic biorthogonal approach to molecular excitation energies, transition probabilities, and excited state properties, J. Chem. Phys. 98, 7029 (1993).

[10] R. J. Bartlett, Coupled-cluster theory and its equationof-motion extensions, WIREs: Comput. Mol. Sci. 2, 126 (2012).

[11] K. Sneskov and O. Christiansen, Excited state coupled cluster methods, WIREs: Comput. Mol. Sci. 2, 566 (2012).

[12] S. Coriani and H. Koch, Communication: X-ray absorption spectra and core-ionization potentials within a core-valence separated coupled cluster framework, J. Chem. Phys. 143, 181103 (2015).

[13] S. Coriani and H. Koch, Erratum: "Communication: X-ray absorption spectra and core-ionization potentials within a core-valence separated coupled cluster framework" [J. Chem. Phys. 143, 181103 (2015)], J. Chem. Phys. 145, 149901 (2016).

[14] M. L. Vidal, X. Feng, E. Epifanovski, A. I. Krylov, and S. Coriani, A new and efficient equation-of-motion coupled-cluster framework for core-excited and coreionized states, J. Chem. Theory Comput. 15, 3117 (2019).

[15] M. L. Vidal, A. I. Krylov, and S. Coriani, Dyson orbitals within the fc-CVS-EOM-CCSD framework: theory and application to X-ray photoelectron spectroscopy of ground and excited states, Phys. Chem. Chem. Phys. 22, 2693 (2020).

[16] R. Faber and S. Coriani, Resonant inelastic X-ray scattering and nonesonant X-ray emission spectra from coupled-cluster (damped) response theory, J. Chem. Theory Comput. 15, 520 (2019).

[17] K. D. Nanda, M. L. Vidal, R. Faber, S. Coriani, and A. I. Krylov, How to stay out of trouble in RIXS calculations within the equation-of-motion coupled-cluster damped response theory framework? Safe hitchhiking in the excitation manifold by means of core-valence separation, Phys. Chem. Chem. Phys. 22, 2629 (2020).

[18] R. Faber and S. Coriani, Core-valence-separated coupled-cluster-singles-and-doubles complexpolarization-propagator approach to X-ray spectroscopies, Phys. Chem. Chem. Phys. 22, 2642 (2020).

[19] K. D. Nanda and A. I. Krylov, A simple molecular orbital picture of RIXS distilled from many-body damped response theory, J. Chem. Phys. 152, 244118 (2020).

[20] K. D. Nanda and A. I. Krylov, Cherry-picking resolvents: A general strategy for convergent coupled-cluster damped response calculations of core-level spectra, J. Phys. Chem. 153, 141104 (2020).

[21] M. L. Vidal, P. Pokhilko, A. I. Krylov, and S. Coriani, Equation-of-motion coupled-cluster theory to model ledge x-ray absorption and photoelectron spectra, J. Phys. Chem. Lett. (2020), in press.

[22] R. Sarangi, M. L. Vidal, S. Coriani, and A. I. Krylov, On the basis set selection for calculations of core-level states: Different strategies to balance cost and accuracy, Mol. Phys. 118, e1769872 (2020).

[23] Z.-H. Loh, G. Doumy, C. Arnold, L. Kjellsson, S. H. Southworth, A. Al Haddad, Y. Kumagai, M.-F. Tu, P. J. Ho, A. M. March, R. D. Schaller, M. S. Bin Mohd Yusof, T. Debnath, M. Simon, R. Welsch, L. Inhester, K. Khalili, K. D. Nanda, A. I. Krylov, S. Moeller, G. Coslovich, J. Koralek, M. P. Minitti, W. F. Schlotter, J.-E. Rubensson, R. Santra, and L. Young, Observation of the fastest chemical processes in the radiolysis of water, Science 367, 179 (2020).

[24] L. Kjellsson, K. D. Nanda, J.-E. Rubensson, G. Doumy, S. H. Southworth, P. J. Ho, A. M. March, A. Al Haddad, Y. Kumagai, M.-F. Tu, T. Debnath, M. S. Bin Mohd Yusof, C. Arnold, W. F. Schlotter, S. Moeller, G. Coslovich, J. D. Koralek, M. P. Minitti, M. L. Vidal, M. Simon, R. Santra, Z.-H. Loh, S. Coriani, A. I. Krylov, and L. Young, Resonant inelastic x-ray scattering reveals hidden local transitions of the aqueous $\mathrm{OH}$ radical, Phys. Rev. Lett. 124, 236001 (2020).

[25] M. L. Vidal, M. Epshtein, V. Scutelnic, Z. Yang, T. Xue, S. R. Leone, A. I. Krylov, and S. Coriani, The interplay of open-shell spin-coupling and Jahn-Teller distortion in benzene radical cation probed by x-ray spectroscopy, J. Phys. Chem. A (2020), submitted; https://doi.org/10.26434/chemrxiv.12799922.v1.

[26] W. Skomorowski and A. I. Krylov, Feshbach-fano approach for calculation of Auger decay rates using equation-of-motion coupled-cluster wave functions: Theory and implementation, J. Chem. Phys. (2020).

[27] J. V. Ortiz, Dyson-orbital concepts for description of electrons in molecules, J. Chem. Phys. 153, 070902 (2020).

[28] A. I. Krylov, From orbitals to observables and back, J. Chem. Phys. 153, 080901 (2020).

[29] R. Krishnan, J.S. Binkley, R. Seeger, and J.A. Pople, Self-consistent molecular orbital methods. XX. A basis set for correlated wave functions, J. Chem. Phys. 72, 650 (1980).

[30] M.J. Frisch, J.A. Pople, and J.S. Binkley, Selfconsistent molecular orbital methods 25. Supplementary functions for Gaussian basis sets, J. Chem. Phys. 
80, 3265 (1984).

[31] M. S. Szczygieł, M. Lesiuk, and R. Moszynski, Theoretical description of the ionization processes with a discrete basis set representation of the electronic continuum, arXiv:1909.07833 (2019).

[32] Y. Shao, Z. Gan, E. Epifanovsky, A.T.B. Gilbert, M. Wormit, J. Kussmann, A.W. Lange, A. Behn, J. Deng, X. Feng, D. Ghosh, M. Goldey, P.R. Horn, L.D. Jacobson, I. Kaliman, R.Z. Khaliullin, T. Kus, A. Landau, J. Liu, E.I. Proynov, Y.M. Rhee, R.M. Richard, M.A. Rohrdanz, R.P. Steele, E.J. Sundstrom, H.L. Woodcock III, P.M. Zimmerman, D. Zuev, B. Albrecht, E. Alguires, B. Austin, G.J.O. Beran, Y.A. Bernard, E. Berquist, K. Brandhorst, K.B. Bravaya, S.T. Brown, D. Casanova, C.-M. Chang, Y. Chen, S.H. Chien, K.D. Closser, D.L. Crittenden, M. Diedenhofen, R.A. DiStasio Jr., H. Do, A.D. Dutoi, R.G. Edgar, S. Fatehi, L. Fusti-Molnar, A. Ghysels, A. Golubeva-Zadorozhnaya, J. Gomes, M.W.D. Hanson-Heine, P.H.P. Harbach, A.W. Hauser, E.G. Hohenstein, Z.C. Holden, T.-C. Jagau, H. Ji, B. Kaduk, K. Khistyaev, J. Kim, J. Kim, R.A. King, P. Klunzinger, D. Kosenkov, T. Kowalczyk, C.M. Krauter, K.U. Laog, A. Laurent, K.V. Lawler, S.V. Levchenko, C.Y. Lin, F. Liu, E. Livshits, R.C. Lochan, A. Luenser, P. Manohar, S.F. Manzer, S.-P. Mao, N. Mardirossian, A.V. Marenich, S.A. Maurer, N.J. Mayhall, C.M. Oana, R. Olivares-Amaya, D.P. O'Neill, J.A. Parkhill, T.M. Perrine, R. Peverati, P.A. Pieniazek, A. Prociuk, D.R. Rehn, E. Rosta, N.J. Russ, N. Sergueev, S.M. Sharada, S. Sharmaa, D.W. Small, A. Sodt, T. Stein, D. Stuck, Y.-C. Su, A.J.W. Thom, T. Tsuchimochi, L. Vogt, O. Vydrov, T. Wang, M.A. Watson, J. Wenzel, A. White, C.F. Williams, V. Vanovschi, S. Yeganeh, S.R. Yost, Z.-Q. You, I.Y. Zhang, X. Zhang, Y. Zhou, B.R. Brooks, G.K.L. Chan, D.M. Chipman, C.J. Cramer, W.A. Goddard III, M.S. Gordon, W.J. Hehre, A. Klamt, H.F. Schaefer III, M.W. Schmidt, C.D. Sherrill, D.G. Truhlar, A. Warshel, X. Xu, A. Aspuru-Guzik, R. Baer, A.T. Bell, N.A. Besley, J.D. Chai, A. Dreuw, B.D. Dunietz, T.R. Furlani, S.R. Gwaltney, C.-P. Hsu, Y. Jung, J. Kong, D.S. Lambrecht, W.Z. Liang, C. Ochsenfeld, V.A. Rassolov, L.V. Slipchenko, J.E. Subotnik, T. Van Voorhis, J.M. Herbert, A.I. Krylov, P.M.W. Gill, and M. Head-Gordon, Advances in molecular quantum chemistry contained in the Q-Chem 4 program package, Mol. Phys. 113, 184 (2015).

[33] A. I. Krylov and P. M. W. Gill, Q-Chem: An engine for innovation, WIREs: Comput. Mol. Sci. 3, 317 (2013).

[34] S. Wolfram, Mathematica, Wolfram Research, Inc.

[35] A. Albiez, M. Thoma, W. Weber, and W. Mehlhorn, $K L-2,3$ ionization in neon by electron impact in the range $1.5-50 \mathrm{keV}$ : cross sections and alignment, $\mathrm{Z}$. Phys. D 16, 97 (1990).

[36] Y. Sakai and T. Anno, New set of rules of the Slater type on the screening constants and the effective principal quantum numbers, J. Chem. Phys. 60, 620 (1974).

[37] A. Müller, D. Bernhardt, A. Borovik Jr, T. Buhr, J. Hellhund, K. Holste, D. Kilcoyne, S. Klumpp, M. Martins, S. Ricz, J. Seltmann, J. Viefhaus, and S. Schippers, Photoionization of $\mathrm{Ne}$ atoms and $\mathrm{Ne}^{+}$ions near the $K$ edge: Precision spectroscopy and absolute cross-sections, Astrophys. J. 836, 166 (2017).

[38] G. Howat, T. Aberg, and O. Goscinski, Relaxation and final-state channel mixing in the Auger effect, J. Phys. B 11, 1575 (1978).

[39] J. Tulkki, T. Åberg, A. Mäntykenttä, and H. Aksela, Relativistic multichannel calculation of the Ne KLL and $\operatorname{Ar} L_{2} M_{2,3} M_{2,3}$ Auger transition rates, Phys. Rev. A 46, 1357 (1992).

[40] M. Leväsalmi, H. Aksela, and S. Aksela, Satellite structure in the KLL spectrum of neon, Phys. Scr. 1992, 119 (1992).

[41] M. Coreno, L. Avaldi, R. Camilloni, KC. Prince, M. De Simone, J. Karvonen, R. Colle, and S. Simonucci, Measurement and ab initio calculation of the ne photoabsorption spectrum in the region of the $K$ edge, Phys. Rev. A 59, 2494 (1999).

[42] S. Svensson, N. Mårtensson, E. Basilier, P. A. Malmquist, U. Gelius, and K. Siegbahn, Lifetime broadening and CI-resonances observed in ESCA, Phys. Scr. 14, 141 (1976).

[43] J.M. Esteva, B. Gauthe, P. Dhez, and R.C. Karnatak, Double excitation in the $K$ absorption spectrum of neon, J. Phys. B 16, L263 (1983).

[44] C.M. Teodorescu, R.C. Karnatak, J.M. Esteva, A. El Afif, and J.-P. Connerade, Unresolvable rydberg lines in x-ray absorption spectra of free atoms, J. Phys. B 26, 4019 (1993).

[45] L. Avaldi, G. Dawber, R. Camilloni, G.C. King, M. Roper, M.R.F. Siggel, G. Stefani, M. Zitnik, A. Lisini, and P. Decleva, Measurement of ne $1 s$ and $1 s n l$ satellite photoelectron spectra near threshold, Phys. Rev. A 51, 5025 (1995).

[46] V. Carravetta, H. Ågren, and A. Cesar, Is the core photoelectron band of neon asymmetric or not?, Chem. Phys. Lett. 180, 358 (1991).

[47] J. C. Slater, Atomic shielding constants, Phys. Rev. 36, 57 (1930).

[48] E. Clementi and D.L. Raimondi, Atomic screening constants from scf functions, J. Chem. Phys. 38, 2686 (1963).

[49] M. Guerra, P. Amaro, J.P. Santos, and P. Indelicato, Relativistic calculations of screening parameters and atomic radii of neutral atoms, At. Data Nucl. Data Tables 117, 439 (2017).

[50] W. E. Moddeman, T. A. Carlson, M. O. Krause, B. P. Pullen, W. E. Bull, and G. K. Schweitzer, Determination of the $K L L$ Auger spectra of $\mathrm{N}_{2}, \mathrm{O}_{2}, \mathrm{CO}, \mathrm{NO}$, $\mathrm{H}_{2} \mathrm{O}$, and $\mathrm{CO}_{2}$, J. Chem. Phys. 55, 2317 (1971).

[51] H. Siegbahn, L. Asplund, and P. Kelfve, The Auger electron spectrum of water vapour, Chem. Phys. Lett. 35, 330 (1975).

[52] H. Ågren and H. Siegbahn, Semi-internal correlation in the auger electron spectrum of $\mathrm{H}_{2} \mathrm{O}$, Chem. Phys. Lett. 69, 424 (1980).

[53] V. Carravetta and H. Ågren, Stieltjes imaging method for molecular Auger transition rates: Application to the Auger spectrum of water, Phys. Rev. A 35, 1022 (1987).

[54] A. Cesar, H. Ågren, and V. Carravetta, Vibronic emission from short-lived core-hole states: Theory and applications for the water molecule, Phys. Rev. A 40, 187 (1989). 
[55] G. Öhrwall, R.F. Fink, M. Tchaplyguine, L. Ojamäe, M. Lundwall, R.R.T. Marinho, A. Naves de Brito, S.L. Sorensen, M. Gisselbrecht, R. Feifel, T. Rander, A. Lindblad, J. Schulz, L. J. Sæthre, N. Mårtensson, S. Svensson, and O. Björneholm, The electronic structure of free water clusters probed by auger electron spectroscopy, J. Chem. Phys. 123, 054310 (2005).

[56] L. Inhester, C. F. Burmeister, G. Groenhof, and H. Grubmueller, Auger spectrum of a water molecule after single and double core ionization, J. Chem. Phys. 136, 144304 (2012).

[57] Y. Hori, M. Nishida, F. H. Lim, T. Ida, and M. Mizuno, Simulation of molecular Auger spectra using a twoelectron Dyson propagator, J. Electron. Spectrosc. Relat. Phenom. 207, 60 (2016).

[58] R. Sankari, M. Ehara, H. Nakatsuji, Y. Senba, K. Hosokawa, H. Yoshida, A. De Fanis, Y. Tamenori, S. Aksela, and K. Ueda, Vibrationally resolved O $1 s$ photoelectron spectrum of water, Chem. Phys. Lett. 380, 647 (2003).

[59] A. Kivimäki, M. Neeb, B. Kempgens, H. M. Köppe, and A. M. Bradshaw, The C $1 s$ Auger decay spectrum of the molecule: the effects of vibrational fine structure, double excitations and shake-up transitions, J. Phys. B 29, 2701 (1996).

[60] R. Spohr, T. Bergmark, N. Magnusson, L.O. Werme, C. Nordling, and K. Siegbahn, Electron spectroscopic investigation of auger processes in bromine substituted methanes and some hydrocarbons, Phys. Scr. 2, 31 (1970).

[61] R. R. Rye, D. R. Jennison, and J. E. Houston, Auger spectra of alkanes, J. Chem. Phys. 73, 4867 (1980).

[62] F. P. Larkins, L. C. Tulea, and E. Z. Chelkowska, Auger electron spectra of molecules: the first row hydrides, Australian J. Phys. 43, 625 (1990).

[63] L. Asplund, U. Gelius, S. Hedman, K. Helenelund, K. Siegbahn, and P.E.M. Siegbahn, Vibrational structure and lifetime broadening in core-ionised methane, J. Phys. B 18, 1569 (1985).

[64] P.A. Heimann, L.J. Medhurst, M.R.F. Siggel, D.A. Shirley, C.T. Chen, Y. Ma, and F. Sette, Zero electron kinetic energy photoemission of $\mathrm{CH}_{4}$ and $\mathrm{CD}_{4}$ at the carbon $K$ ionization threshold, Chem. Phys. Lett. 183, 234 (1991).

[65] H.M. Köppe, B.S. Itchkawitz, A.L.D. Kilcoyne, J. Feldhaus, B. Kempgens, A. Kivimäki, M. Neeb, and A.M. Bradshaw, High-resolution c $1 s$ photoelectron spectra of methane, Phys. Rev. A 53, 4120 (1996).

[66] T. X. Carroll, N. Berrah, J. Bozek, J. Hahne, E. Kukk, L. J. Sæthre, and T. D. Thomas, Carbon $1 s$ photoelectron spectrum of methane: Vibrational excitation and core-hole lifetime, Phys. Rev. A 59, 3386 (1999).

[67] A. Hiltunen, S. Aksela, Gy. Vıkor, S. Ricz, Á. Kövér, and B. Sulik, High resolution carbon and oxygen $K-L L$ Auger spectra of carbon dioxide, Nucl. Instr. and Meth. in Phys. Res. B 154, 267 (1999).

[68] J. A. Kelber, D. R. Jennison, and R. R. Rye, Analysis of the Auger spectra of $\mathrm{CO}$ and $\mathrm{CO}_{2}$, J. Chem. Phys. 75, 652 (1981).

[69] H. Agren, On the interpretation of molecular valence auger spectra, J. Chem. Phys. 75, 1267 (1981).
[70] J. H. D. Eland, S. Plogmaker, P. Lablanquie, F. Penent, J. Palaudoux, C. Nicolas, E. Robert, C. Miron, and R. Feifel, Wide range double photoionisation spectra of $\mathrm{N}_{2}$ and $\mathrm{CO}_{2}$, Chem. Phys. Lett. 646, 31 (2016).

[71] V. Feyer, P. Bolognesi, M. Coreno, K.C. Prince, L. Avaldi, L. Storchi, and F. Tarantelli, Effects of nuclear dynamics in the low-kinetic-energy auger spectra of $\mathrm{CO}$ and $\mathrm{CO}_{2}$, J. Chem. Phys. 123, 224306 (2005).

[72] R. Püttner, V. Sekushin, G. Kaindl, X.J. Liu, H. Fukuzawa, K. Ueda, T. Tanaka, M. Hoshino, and H. Tanaka, A vibrationally resolved $\mathrm{C} 1 \mathrm{~s}^{-1}$ auger spectrum of $\mathrm{CO}_{2}$, J. Phys. B 41, 045103 (2008).

[73] W Persson, The spectrum of singly ionized neon, Ne II, Phys. Scr. 3, 133 (1971).

[74] A. Kivimäki, S. Heinäsmäki, M. Jurvansuu, S. Alitalo, E. Nõmmiste, H. Aksela, and S. Aksela, Auger decay at the $1 s-1 n p(n=3-5)$ resonances of Ne, J. Electron. Spectrosc. Relat. Phenom. 114, 49 (2001).

[75] G. Turri, G. Battera, L. Avaldi, R. Camilloni, M. Coreno, A. Ruocco, R. Colle, S. Simonucci, and G. Stefani, Correlation effects in auger cascade studied by angle resolved coincidence electron spectroscopy: the $1 s \rightarrow 3 p$ excitation in neon, J. Electron. Spectrosc. Relat. Phenom. 114, 199 (2001).

[76] Y. Tamenori and I. H. Suzuki, Multiplet exchange Auger transitions following resonant Auger decays in Ne 1s photoexcitation, J. Phys. B 47, 145001 (2014).

[77] S. Stock, R. Beerwerth, and S. Fritzsche, Auger cascades in resonantly excited neon, Phys. Rev. A 95, 053407 (2017).

[78] H. Aksela, S. Aksela, J. Tulkki, T. Åberg, G. M. Bancroft, and K. H. Tan, Auger emission from the resonantly excited $1 s^{1} 2 s^{2} 2 p^{6} 3 p$ state of Ne, Phys. Rev. A 39, 3401 (1989).

[79] G. Grell, O. Kühn, and S. I. Bokarev, Multireference quantum chemistry protocol for simulating autoionization spectra: Test of ionization continuum models for the neon atom, Phys. Rev. A 100, 042512 (2019).

[80] M. Schmidbauer, A. L. D. Kilcoyne, H.-M. Köppe, J. Feldhaus, and A. M. Bradshaw, Shape resonances and multielectron effects in the core-level photoionization of $\mathrm{CO}_{2}$, Phys. Rev. A 52, 2095 (1995).

[81] T. Porwol, G. Illing, H.-J. Freund, H. Kuhlenbeck, M. Neumann, S. Bernstorff, W. Braun, W. von Niessen, and C.-M. Liegener, Autoionization versus photoionization of molecular adsorbates: $\mathrm{CO}_{2}$ physisorbed on Ni(110), Phys. Rev. B 41, 10510 (1990).

[82] T. X. Carroll and T. D. Thomas, Deexcitation electron spectroscopy of core-excited $\mathrm{CO}_{2}$ : Comparison of experiment with theory, J. Chem. Phys. 94, 11 (1991).

[83] P. Morin, M. Simon, C. Miron, N. Leclercq, E. Kukk, J.D. Bozek, and N. Berrah, Role of bending in the dissociation of selective resonant inner-shell excitation as observed in $\mathrm{CO}_{2}$, Phys. Rev. A 61, 050701 (2000).

[84] E. Kukk, J. D. Bozek, and N. Berrah, Photoexcitation and Auger decay of the Renner-Teller split C $1 s \rightarrow \pi_{u}^{*}$ state in $\mathrm{CO}_{2}$, Phys. Rev. A 62, 032708 (2000).

[85] E. Antonsson, M. Patanen, C. Nicolas, S. Benkoula, J. J. Neville, V. L. Sukhorukov, J. D. Bozek, P. V. Demekhin, and C. Miron, Dynamics of the $\mathrm{C} 1 s \rightarrow \pi^{*}$ excitation and decay in $\mathrm{CO}_{2}$ probed by vibrationally and angularly resolved Auger spectroscopy, Phys. Rev. 
A 92, 042506 (2015).

[86] D. A. Matthews and J. F. Stanton, A new approach to approximate equation-of-motion coupled cluster with triple excitations, J. Chem. Phys. 145, 124102 (2016).
[87] T. C. Jagau, Non-iterative triple excitations in equation-of-motion coupled-cluster theory for electron attachment with applications to bound and temporary anions, J. Chem. Phys. 148, 024104 (2018). 